\title{
Ein Impuls, Resonanz oder Freiheit - was begründet kritische Gesellschaftstheorie?
}

\author{
Sven Ellmers
}

Online publiziert: 6. April 2020

(C) Der/die Autor(en) 2020

Zusammenfassung Die gegenwärtige Debatte um die normativen Grundlagen kritischer Gesellschaftstheorie weist eine große Bandbreite ethischer Positionen auf: vom (meta-)ethischen Negativismus, der gänzlich auf einen positiven Kritik-Maßstab verzichtet, über Entfremdungs- und Resonanztheorien, die zumindest eine grobe Vorstellung des guten Lebens vermitteln wollen, bis hin zu deontologischen Ansätzen, die Gesellschaftskritik in intelligibler Freiheit begründet sehen. Der folgende Beitrag zeigt, dass diese drei Grundpositionen sich mit Problemen konfrontiert sehen, die der hegelsche Freiheitsbegriff zu lösen vermag.

Schlüsselwörter Kritische Theorie $\cdot$ Metaethischer Negativismus · Immanuel Kant · Georg Wilhelm Friedrich Hegel · Freiheit

\begin{abstract}
The current debate about the normative foundations of critical social theory shows a range of ethical positions: from the meta-ethical negativism, which completely dispenses with a positive criterion for critique, to theories of alienation and resonance, which give at least a rough idea of the good life, right down to deontological approaches, which argue that social criticism has to be based on intelligible freedom. The following article shows that these three basic positions face problems that the Hegelian concept of freedom can solve.
\end{abstract}

Keywords Critical Theory · Meta-ethical Negativism · Immanuel Kant · Georg Wilhelm Friedrich Hegel · Freedom

\footnotetext{
S. Ellmers $(\triangle)$

Institut für Philosophie, Fakultät IV, Carl von Ossietzky Universität Oldenburg, 26111 Oldenburg, Deutschland

E-Mail: sven.ellmers@uol.de
} 
Folgt man Jürgen Habermas, hat sich die erste Generation der Frankfurter Schule mit ihrer Dialektik der Aufklärung jeder Möglichkeit beraubt, die normativen Grundlagen ihrer eigenen Kritik ausweisen zu können: Max Horkheimer und Theodor W. Adorno hätten in ihrer Genealogie instrumenteller Naturbeherrschung - von der frühen Mythologie über den Kapitalismus bis zur Shoa - die Vernunft und ihre Institutionalisierungen derart radikal denunziert, dass sie nicht länger den Ort angeben konnten, von dem aus sie ihre Kritik formulierten (vgl. Habermas 1985). Diese Kritik war einschneidend. Zwar konnte insbesondere Adorno kaum abgesprochen werden, sich auch nach der Dialektik der Aufklärung intensiv mit moralphilosophischen Fragen auseinandergesetzt zu haben, insgesamt verfestigte sich jedoch der Verdacht, dass die Begründer der Kritischen Theorie an einem Problem gescheitert waren, das bereits Marx und Engels mit sich herumtrugen: Beide brandmarkten Moral und Moralphilosophie immer wieder als herrschaftslegitimierend, ließen selbst jedoch weitgehend offen, welche normativen Grundüberzeugungen hinter den von ihnen verwendeten dichten ethischen Begriffen stehen, die schon als solche eine Ablehnung des Kapitalismus anzeigen. Vor dem zeithistorischen Hintergrund des sowjetischen Autoritarismus konnte dieses Begründungsdefizit der Klassiker und ihrer Nachfolger nicht länger ignoriert werden; es verschob sich zunehmend der Fokus von der Ausübung der Kritik hin zu ihrer ethischen Fundierung.

Der Herausforderung, die Habermas für Verfechter der älteren Kritischen Theorie darstellt, ist auf ganz unterschiedliche Weisen begegnet worden. Eher selten sind die Versuche, sich intensiv mit der formalpragmatischen Antwort zu beschäftigen, die Habermas auf die von ihm aufgeworfene Frage gab. Axel Honneth (2000) etwa erhob zwar den hermeneutischen Einwand, dass die Diskursethik den Erfahrungen wirklicher Akteure nicht Rechnung trage - Protest und Widerstand seien das Resultat einer empfundenen Missachtung sozialisatorisch erworbener Identitätsansprüche, nicht der Verletzung eines abstrakten, aus unbewusst beherrschten Sprachregeln destillierten Moralprinzips -, aber eine eingehende Prüfung ihrer Prämissen und Konsistenz blieb aus. Diese Aufgabe übernahmen meist Vertreter der analytischen Philosophie (Steinhoff 2006; Lumer 1997).

Ein Grund dafür ist sicherlich, dass einige Autoren, die sich der kritischen Theorie verpflichtet fühlen, bereits die von Habermas aufgeworfene Frage zurückweisen. Es sei, so argumentiert beispielsweise Fabian Freyenhagen für einen Metaethischen Negativismus (I), weder erforderlich noch möglich, einen positiven ethischen Maßstab anzugeben, um mit den bestehenden Verhältnissen ins Gericht zu gehen. Das Begründungsdefizit der alten Kritischen Theorie sei gar kein Defizit, sondern sachlich geboten.

Die meisten Autoren favorisieren jedoch eine andere Strategie. Sie teilen die Ansicht, einen Maßstab (oder mehrere Maßstäbe) der Kritik angeben zu müssen, ersetzen jedoch das Universalisierungsprinzip, das Habermas aus den Präsuppositionen der Rede zu gewinnen können glaubte, durch eine alternative Metanorm. Einigkeit besteht allerdings weder im Hinblick auf die Norm selbst noch im Hinblick auf das methodische Verfahren, sie zu identifizieren.

So vertreten Honneth (2011), Jaeggi (2014, 261-309) und Stahl (2013) die Ansicht, dass die Normen der Kritik noch ihrem Gegenstand zu entnehmen sind. Einen 
externen Maßstab lehnen sie insofern ab. Methodisch ähnelt ihr Plädoyer für reine Normimmanenz der Ideologiekritik des frühen Habermas. Dieser konfrontierte 1962 in Strukturwandel der Öffentlichkeit das bürgerliche Ideal demokratischer Öffentlichkeit mit der bürgerlichen Wirklichkeit. Als er sein Buch 28 Jahre später erneut herausgab, stellte er rückblickend jedoch fest, dass es auf ,geschichtsphilosophischen Hintergrundannahmen“ beruhte und somit der Kontingenz von Wertvorstellungen nichts entgegenzusetzen hatte: ,Wenn die bürgerlichen Ideale eingezogen werden, wenn das Bewußtsein zynisch wird, verfallen jene Normen und Wertorientierungen, für die die Ideologiekritik Einverständnis voraussetzen muss, wenn sie daran appellieren will. Ich habe deshalb vorgeschlagen, die normativen Grundlagen der kritischen Gesellschaftstheorie tiefer zu legen." (Habermas 1990, 34). Das Problem, das eine rein immanente Kritik sich auf einen normativen Überschuss der Gesellschaft verlassen können muss, die sie doch zu kritisieren beabsichtigt, zeigt sich u.a. in Honneths normativer Rekonstruktion des Marktes: Honneth muss am Ende selbst eingestehen, dass die neoliberalen Transformationsprozesse der letzten Jahrzehnte sich nicht in der Neuausrichtung wirtschaftlicher und staatlicher Institutionen erschöpfen, sondern auch einen grundlegenden Mentalitätswandel zur Folge hatten - der wiederum erklärt, warum der Abbau sozialer Errungenschaften keine nennenswerte Proteste mehr hervorruft. Es ist der Kritik-Modus reiner Normimmanenz, der Honneth am Ende ,in Verlegenheit“ (2011, 460) bringt.

Dass sich immanente Kritiken von der Verfasstheit ihres Gegenstands abhängig machen, kann, muss sich jedoch nicht als ein erheblicher Nachteil erweisen. ${ }^{1}$ Schon weil der Fokus zuletzt etwas einseitig auf ihnen lag, möchte ich jedoch andere Beiträge zur Normativitätsdebatte in den Mittelpunkt rücken. Nach der Darstellung des Metaethischen Negativismus werde ich zunächst auf die Entfremdungs- bzw. Resonanztheorie von Hartmut Rosa eingehen (II), bevor ich Frank Kuhnes These diskutiere, dass kritische Gesellschaftstheorie ohne den kategorischen Imperativ Kants nicht denkbar sei (III). Auf die perfektionistische Tradition komme ich im Rahmen des letzten Teils zu sprechen (IV).

Damit deutet sich schon an, dass die Debatten innerhalb der kritischen Theorie ähnlich vielstimmig sind wie in der normativen Ethik selbst - lediglich utilitaristische Positionen spielen in ihr keine Rolle. Anders als MacIntyre (1995), der den modernen moralphilosophischen Diskurs für endlos erachtet, weil die Prämissen der widerstreitenden Positionen im Rahmen unserer emotivistischen Kultur nichts weiter als subjektive Überzeugungen darstellten, bin ich der Auffassung, dass sich die gegenwärtige Debatte auf Grundlage der Aufklärungsphilosophie argumentativ führen lässt. Dafür bedarf es allerdings eines ethischen Prinzips, welches in der Lage ist, der Vielfalt normativer Maßstäbe Rechnung zu tragen, genauer gesagt sie auf nicht-eklektizistische Weise in sich zu integrieren. Dass der Freiheitsbegriff Hegels genau dies leistet, werde ich im letzten Teil des Beitrags zeigen.

\footnotetext{
1 Zumal sich das angesprochene Problem zumindest eindämmen lässt: So sind die Wertvorstellungen, die Jaeggi als Bezugspunkt für ihre immanente Kritik der modernen Ökonomie dienen, nicht kontingenter Natur, sondern gehören zum Kernbestand bürgerlicher Gesellschaften.
} 


\section{Metatethischer Negativismus}

Ein erheblicher Teil der gegenwärtigen Debatte ist durch eine tiefsitzende Skepsis gegenüber Letztbegründungen geprägt. Man gibt sich bescheiden. Ähnlich wie John Rawls (2001), der davon ausging, dass die burdens of judgment selbst bei vernünftig argumentierenden Personen zu inkompatiblen Weltanschauungen führen, weshalb ein overlapping consensus nur erreichbar sei, wenn der Gegenstandsbereich der Gerechtigkeitstheorie (auf die politische Grundstruktur der Gesellschaft) eingeschränkt und auf bezweifelbare metaphysische Annahmen verzichtet werde, plädieren auch viele Debattenbeiträge in der kritischen Theorie für Enthaltsamkeit, sobald die Frage nach der Wahrheit letzter ethischer Geltungsansprüche im Raum steht.

Besonders weitgehend ist hier der metaethische Negativismus von Fabian Freyenhagen $(2017,2013)$, dessen entscheidender Gewährsmann Adorno ist. Adorno habe nämlich nicht nur einen ,methodischen Negativismus“ vertreten, der mit den zwischenmenschlichen Pathologien und Defizienzerfahrungen beginne und diese anschließend erkläre, sondern auch einen „epistemischen Negativismus“ (Freyenhagen 2017, 230). Demzufolge können wir zwar erkennen, dass die sozialen Verhältnisse eine Verwirklichung menschlicher Potentiale verhindern, ja dass sie uns geradezu seelisch verkrüppeln, jedoch lasse sich nicht positiv angeben, was genau dieses Potential sei und wie folglich eine bessere Gesellschaft im Detail einzurichten wäre jede Utopie wäre noch von der abzulehnenden Gegenwart affiziert (vgl. ebd., 230). Wir können also wohl angeben, was schlecht, nicht aber, was gut ist.

Wie schon Adorno changiert auch Freyenhagen zwischen einem rigorosen Bilderverbot - über eine postkapitalistische Gesellschaft lasse sich buchstäblich nichts sagen - und dem moderateren Appell, keine allzu konkreten Utopien zu entwerfen. Eine vergleichbare Unentschiedenheit begegnet uns in Freyenhagens Ausführungen zum normativen Kriterium der Gesellschaftskritik. Einerseits werden von ihm (unter Rekurs auf Adorno) en passant mehrmals normative Kriterien verwendet: Es ist von der Verwirklichung eines unerfüllten menschlichen Potentials die Rede (vgl. ebd., 229f.) - was bei Adorno auf eine perfektionistische, an Aristoteles anknüpfende Tradition verweist ${ }^{2}$-, es wird von einer ,befreiten Menschheit“" gesprochen (ebd., 231) oder es wird die Verhinderung von „Leid und Unrecht“ (ebd., 235) als Ziel angegeben. Andererseits lehnt Freyenhagen die Aufforderung von Habermas, sich über die normativen Grundlagen der eigenen Kritik Rechenschaft abzulegen, aus folgenden Gründen rundweg ab:

1. Die Erwartung, die normativen Geltungsansprüche der kritischen Theorie zu begründen, laufe auf ein unkritisches Verhältnis zum Gegenstand der Kritik hinaus: ,anzunehmen, dass das Recht auf Kritik erst zu verdienen sei, setzt voraus, dass der Status quo erst einmal als Standard anerkannt wird, von dem Abweichungen gerechtfertigt werden müssten. [...] Warum ist angesichts der langen Menschheitsgeschichte von Unterdrückung und Elend und dem heutigen Fortbestehen dieser Übel nicht die gegenteilige Position - dass wir das Recht am Festhalten am Status quo verdienen

\footnotetext{
2 Besonders deutlich wird dies in der kleineren Schrift „Freizeit“, in der Adorno (1997a) vorsichtig zu erkennen gibt, wie das Verhältnis von Arbeit und freier Zeit in einer postkapitalistischen Gesellschaft aussehen könnte.
} 
müssen - angemessener?“ (ebd., 233f.) Sicher, die Befürworter der gegenwärtigen Gesellschaftsstruktur stehen argumentativ ebenfalls in der Bringschuld - nur entbindet dies die kritische Theorie von ihrer? Die Forderung, die normativen Grundlagen der eigenen Theorie offenzulegen, schließt eine an die theoretischen Kontrahenten gerichtete Forderung logisch keineswegs aus, ihrerseits gute Gründe für die Beibehaltung des status quo anzugeben. Darüber hinaus wird das, was Freyenhagen wie ein disjunktives Oder präsentiert, weder dem Ansinnen von Habermas noch der Theoriegeschichte der bürgerlichen Gesellschaft gerecht: War die Etablierung und Ausweitung einer staatlich flankierten Privateigentumsordnung doch stets begleitet von philosophischen Entwürfen, die diese Entwicklung auch normativ rechtfertigten. Im direkten Vergleich, hierin wird man Habermas zustimmen müssen, gab es auf Seiten der Arbeiterbewegung und linken Theorie nur wenige Entsprechungen.

2. Die Forderung, moralische Urteile immer auch begründen zu können, weist Freyenhagen zurück, weil sie nicht nur das Resultat einer geistigen Reflexion darstellen, sondern auf einen somatischen Impuls zurückgehen (ebd., 234f.). Vorbild ist hier wiederum Adorno:

Hitler hat den Menschen im Stande ihrer Unfreiheit einen neuen kategorischen Imperativ aufgezwungen: ihr Denken und Handeln so einzurichten, daß Auschwitz nicht sich wiederhole, nichts Ähnliches geschehe. Dieser Imperativ ist so widerspenstig gegen seine Begründung wie einst die Gegebenheit des Kantischen. Ihn diskursiv zu behandeln, wäre Frevel: an ihm läßt leibhaft das Moment des Hinzutretenden am Sittlichen sich fühlen. Leibhaft, weil es der praktisch gewordene Abscheu vor dem unerträglichen physischen Schmerz ist [...]. (Adorno 1997b, 358)

Wer verlange, dass der neue kategorische Imperativ ausgehend von den Prinzipien einer abstrakten Ethik begründbar sein müsse, begehe einen Frevel; dass millionenfacher Mord sich niemals wieder ereigne, bedürfe keiner gesonderten theoretischen Rechtfertigung, sondern sei schlichtweg eine impulsartige Reaktion (die uns von einer Zivilisation instrumenteller Naturbeherrschung ausgetrieben wurde). Das Problem: Selbst wenn wir Adornos Überlegungen zum neuen kategorischen Imperativ für überzeugend hielten, wären sie ungeeignet, das von Habermas angestoßene Projekt einer normativen Begründung kritischer Theorie auszuhebeln. Dies wäre lediglich dann der Fall, wenn folgende Bedingungen erfüllt wären.

a) Die von Habermas aufgeworfene Fragestellung wäre tatsächlich gegenstandslos, wenn Moralität nicht nur ein irreduzibles somatisches Moment aufwiese, sondern jedes moralische Urteil auch darauf reduzierbar wäre: Über normative Gründe zu reflektieren wäre reine Zeitverschwendung (pragmatisch sinnlos), wäre der Mensch in moralischer Hinsicht nicht mehr als ein Automat.

b) Die begründungsbefreite, spontan-somatische Reaktion als normativer Minimalmaßstab kritischer Theorie kann sich sinnvollerweise nicht auf ein subjektives Leiden beziehen. Subjektiv empfundenes Leid ist offen für willkürliche Bestimmungsgründe, damit nicht verallgemeinerbar und als normatives Kriterium untauglich. Es ließe sich nicht nur gegen bestimmte (heteronom verfasste) Gesellschaften, sondern grundsätzlich gegen alle nur denkbaren Gemeinwesen in Anschlag bringen: Wer beispielsweise daran leidet, kein Multimilliardär sein/werden zu können, wird 
in einer postkapitalistischen Gesellschaft nicht leben wollen. Der somatische Impuls kann also nur ein solcher sein, der sich bei der Verletzung grundlegender Interessen einstellt (wie bei Mord, bei Folter oder Hunger).

c) Schließlich müsste der Nachweis erbracht werden, dass bürgerlich-kapitalistische Gesellschaften zwangsläufig ein solches objektives Leid hervorbringen, das heißt auch alle zukünftigen Reformen und Entwicklungen daran nichts zu ändern vermögen. Der metaethische Negativismus mag attraktiv erscheinen, weil er den moralphilosophischen Argumentationsballast abwerfen zu können glaubt, bürdet sich damit jedoch einen (gesellschafts-)analytischen Nachweis auf, den er wohl schwerlich erbringen können wird.

Wie wir gesehen haben, versucht Freyenhagen in einem ersten Schritt den moralphilosophischen Begründungsprojekten von Habermas et al. den Boden zu entziehen. In einem zweiten, defensiven Schritt verteidigt er Adornos Negativismus gegen einige Einwände, die bereits erhoben wurden oder erhoben werden könnten.

1. Ein Einwand könne lauten, dass ,die Tatsache, dass etwas schlecht ist, uns nicht von sich aus begründet, es zu ändern oder zu kritisieren. Das Schlechte, so könnte gedacht werden, ist nicht unabhängig normativ; wir haben nur Gründe zu handeln (oder zu kritisieren), wenn wir auch auf das Gute verweisen“" (Freyenhagen 2017, 237). Freyenhagen hält diesen Einwand für abwegig: „Nehmen wir das Beispiel des Schmerzes; wenn wir Schmerz empfinden, wollen wir, dass er vorübergeht [...]. In diesem Sinne gibt uns die Negativität des Schmerzes (seine Schmerzhaftigkeit) einen Grund, ihn zu vermeiden, wofür kein Appell an das Gute notwendig zu sein scheint." (ebd.)

Aber, der Einwand liegt auf der Hand, besteht das Gute in diesem Fall nicht in der Abwesenheit des Schmerzes? Dies ist es doch, was die Schmerz empfindende Person herbeisehnt: dass es aufhört. Das Freisein von Schmerz ist zum einen intrinsisch gut, zum anderen handelt es sich um ein transzendentales Gut: Es ist die Bedingung der Möglichkeit, individuelle Interessen auszubilden und zu verfolgen (ähnlich wie das Gut Sicherheit: Nur wer nicht ständig um sein Leben fürchten muss, kann ihm eine mittel- bis langfristige Richtung geben (vgl. Kersting 2000, 26ff.; Stemmer 2000, 195-198)).

2. Freyenhagen kennt diesen Einwand ebenfalls, weshalb er sich bemüht, ihn durch eine begriffliche Unterscheidung zu entkräften: „Die Abwesenheit des Schlechten zeigt [...] einen neutralen Zustand an, noch nicht einen guten." (Freyenhagen 2017, 240) Ein Wissen um das Schlechte impliziere lediglich ein Wissen darüber, wie das Gute nicht beschaffen sei, nicht aber, wie es in positiver Hinsicht beschaffen sei. „Es könnte zum Beispiel mehr als eine Gesellschaftsordnung geben, die verhindert, dass sich Ereignisse wie Auschwitz wiederholen, und selbst wenn es nur eine solche Ordnung gibt, haben wir aus dem Wissen, wie sie in dieser einen Hinsicht nicht sein wird, substantiell wenig über sie erfahren." (ebd.)

Ja, aber liegt nicht gerade darin das Problem? Die Suche nach einem normativen Maßstab der Gesellschaftskritik ist doch insbesondere dadurch motiviert, sich die impliziten Kriterien der Kritik bewusst zu machen (und möglicherweise zu verändern), um davon ausgehend eine zumindest grobe Vorstellung zu gewinnen, wie es anders sein könnte. Ein moralischer Minimalismus schließt zwar inhumane Verbrechen aus, schließt aber auch vieles ein, was ethisch ebenfalls abzulehnen wäre. 
Das Argument, man wolle zukünftigen Generationen nicht durch eine zu detaillierte Utopie vorgreifen, sie nicht in ihrer Freiheit einschränken, mag im Hinblick auf zahlreiche politische Konzeptionen zutreffend sein, greift jedoch nicht beim Projekt einer normativen Fundierung kritischer Theorie: Geht es hier doch um das ethische Grundprinzip, das heißt um den letzten Maßstab aller konkreten Maßstäbe. Wer sich jedoch außerstande sieht, selbst zur letzten Norm der konkreten Normen etwas halbwegs Gehaltvolles zu sagen, sagt nicht besonders viel: weder über das, was an der kapitalistischen Gesellschaft anstößig ist, noch darüber, welchen Kriterien eine alternative Gesellschaft genügen müsste.

3. Schließlich geht Freyenhagen auf den möglichen Einwand ein, nur eine konkrete Vorstellung vom guten Leben könne die Menschen motivieren, sich politisch zu engagieren. Dem hält er entgegen, dass positive Utopien für die Menschen ,wahrscheinlich abstrakter als die konkreten Übel sind, die den Menschen nur zu oft gut vertraut sind." (ebd., 247) Nicht die vage Idee der Gerechtigkeit motiviere, sondern der Zorn über erlittenes Unrecht.

Dies mag motivationspsychologisch richtig sein, übersieht aber den entscheidenden Punkt: Dass eine Verteilung von Gütern oder sozialen Positionen als ungerecht und empörend empfunden werden kann, setzt einen zumindest impliziten Gerechtigkeitssinn voraus. Sobald wir das Terrain des moralischen Minimalismus überschreiten, uns also nicht mehr auf Verbrechen gegen die Menschlichkeit beschränken, vermag die Grundidee des epistemischen Negativismus nicht länger zu überzeugen: Das Wissen um das Schlechte ist nicht geltungsunabhängig von der Idee des Guten. Oder positiv gewendet: Erst im Lichte unserer Vorstellungen vom Guten sind wir in der Lage, negative Evaluationen vorzunehmen. Und da diese häufig impliziten Vorstellungen durch vorreflexive soziale Routinen bedingt sind, erfüllt die sie uns vor Augen führende und überprüfende normative Ethik eine unerlässliche Korrektivfunktion. Die Frage nach dem guten und gelingenden Leben bedarf einer Antwort.

\section{Entfremdungs- und Resonanztheorie}

Die besonderen Gegenstände der Sozialforschung, so Hartmut Rosa, sind letztlich dadurch motiviert, die Bedingungen zu identifizieren, die menschliche Lebensführung gelingen oder scheitern lassen. Er wendet sich zwar einerseits gegen eine ahistorische Wesensdefinition des Menschen - ,sie wird unvermeidlich paternalistisch, wenn sie sich gegenüber den handelnden Subjekten im Besitz der Kenntnis über die ,wahre Natur" oder die ,wahren Bedürfnisse " des Menschen wähnt" (Rosa $2009,91)$-, andererseits dürfe daraus nicht der falsche Schluss gezogen werden, die Frage nach den Bedingungen des guten Leben ausklammern und sich auf das Thema der Verteilungsgerechtigkeit beschränken zu müssen. „Eine Gesellschaft kann vollkommene Verteilungsgerechtigkeit wahren und dennoch von der Austrocknung ihrer Sinnressourcen [...] geprägt sein.“ (ebd.) Rosa hat es sich deshalb zur Aufgabe gemacht, a) in Form einer Beschleunigungsanalyse das Wesensmerkmal der modernen Gesellschaft begrifflich präzise zu bestimmen, b) phänomenologisch darzustellen, wie die Prozesse einer fortlaufenden Dynamisierung subjektiv erfahren werden und 
c) mit seiner Resonanztheorie das normative Gegenstück zu den beschriebenen Entfremdungsphänomenen anzugeben.

a) Eine moderne Gesellschaft zeichnet sich Rosa zufolge dadurch aus, dass sie ihre grundlegenden Institutionen - wie die kapitalistische Marktwirtschaft oder das politische System - sowie ihre sozialstrukturelle Gliederung in Klassen und Schichten nur solange aufrechterhalten kann, wie sie sich fortlaufend transformiert: „Eine Gesellschaft ist modern, wenn sie sich nur (noch) dynamisch zu stabilisieren vermag, wenn sie also systematisch auf Wachstum, Innovationsverdichtung und Beschleunigung angewiesen ist, um ihre Struktur zu erhalten und zu reproduzieren." (Rosa 2017, 335) Anders als viele sozialwissenschaftliche Theorien, die die Moderne als einen Prozess der Individualisierung, Rationalisierung und sozialen Differenzierung beschreiben, lehnt Rosa die Vorstellung ab, die Moderne sei ein normativ zielgerichtetes Projekt: Das Kriterium der dynamischen Stabilisierung könne nicht nur von liberalen, sondern auch von totalitären Gesellschaften erfüllt werden (vgl. ebd., 336). Dies bedeutet im Umkehrschluss nicht, dass außer-moderne Gesellschaften vollkommen statisch gewesen wären oder es sein müssten. In vormodernen Gesellschaften habe es durchaus soziale, kulturelle und ökonomische Veränderungen gegeben, jedoch vollzogen sie sich vor dem Hintergrund veränderter Umweltbedingungen (externe Faktoren wie Kriege, Seuchen, Klimaschwankungen etc.). Im Unterschied zu diesem „adaptiven“ (ebd., 338) Reproduktionsmodus walte in der Moderne eine endogene Eskalationslogik. Zum einen seien die Beschleunigungsprozesse nicht von außen angestoßen, sondern strukturell verankert, zum anderen weise die „Mengensteigerung pro Zeiteinheit“ (ebd., 335) einen exponentiellen Verlauf auf: „Wenn die Steigerungsraten prozentual ungefähr gleich bleiben, nehmen die substantiellen Wachstums- und Veränderungsraten exponentiell [...] zu“" (ebd., 337). Diese eskalatorische Beschleunigung lasse sich nicht nur in der kapitalistischen Ökonomie nachweisen - Rosa verweist hier auf die allgemeine Formel des Kapitals: G-W-G' (ebd., 338) -, sondern auch in anderen gesellschaftlichen Teilbereichen wie der Wissenschaft, Politik und Kultur. Moderne Wissenschaft z. B. tradiert nicht länger das, was als unumstößlich wahr gilt, sondern ist stets darauf aus, neues Wissen zu schaffen und bestehendes Wissen zu vertiefen oder zu falsifizieren. Ein ähnlich endloser Prozess zeichne die moderne Politik aus: Legislaturperiode und Regierungsdauer sind in Verfassungsdemokratien begrenzt, weshalb das politische Personal regelmäßig ausgetauscht oder neu legitimiert werde. Die Volksvertreter sind damit ,gezwungen und zugleich fähig, sensibel auf soziale Bedürfnisse und Veränderungen zu reagieren und damit rasche Anpassungsbewegungen zu vollziehen“ (340), was sich insbesondere in der Dynamik moderner Gesetzgebung zeige. Schließlich begnügen sich auch Kunst, Literatur und Musik nicht länger damit, einen bestehenden künstlerischen Kanon zu verwalten, sondern sind getrieben von der Idee, neue Ausdrucksformen und Techniken zu kreieren, das heißt innovativ zu sein (vgl. ebd., 340f.).

b) Wie die freigesetzte Steigerungslogik subjektiv erfahren wird, hat sich mit der Entwicklung der Moderne - das heißt mit ihrem Beschleunigungsgrad - grundlegend geändert. Von ihren Anfängen bis zur Mitte, teilweise bis zum Ende des 20. Jahrhunderts seien wirtschaftliches Wachstum, neue Technologien und sozialer Wandel als Fortschritt interpretiert worden. Die Erhöhung des Lebensstandards durch effektivere Methoden der instrumentellen Naturbeherrschung, die Vernetzung 
von weit entfernten Erdteilen durch Innovationen im Transport- und Kommunikationswesen und die wachsende Toleranz gegenüber individuellen Lebensentwürfen wurde als eine „Steigerung von Autonomie- und Authentizitätschancen“ (ebd., 342) empfunden. Diese positiven Erwartungen, die die Menschen mit der Moderne verbanden, wurden mittlerweile jedoch tief enttäuscht. Die Steigerungsprozesse dienen nicht länger der Verwirklichung des romantisch-expressivistischen Ideals, sein Leben selbstbestimmt zu gestalten, das heißt ein Leben gemäß relativ-stabiler identitätskonstitutiver Wertvorstellungen zu führen, sondern Identität und Lebensgestaltung werden vor dem Hintergrund eines gestiegenen „Kontingenzbewusstseins“ (Rosa 2012, 229) instrumentalisiert und situativ angepasst, um im kompetitiven Steigerungsspiel noch irgendwie mithalten zu können. Das Hamsterrad dreht sich immer schneller: Die Angst, selbst bei äußerster Kraftanstrengung und Flexibilität dennoch zurückzufallen, greift um sich - eine Situation, die viele Menschen psychisch überfordere und den dramatischen Anstieg von Burnout-Diagnosen erkläre. Wer nicht erkranke, mache im Beruf dennoch die Erfahrung, aufgrund von zunehmendem Zeit- und Optimierungsdruck seine Arbeit nicht mehr so verrichten zu können, wie es eigentlich erforderlich wäre (vgl. Rosa 2017, 354): So muss etwa der Krankenpfleger immer mehr Patienten abarbeiten, anstatt sich ihnen individuell widmen zu können. Subjekte, denen die Welt in erster Linie als äußerer Zwang erscheint, entwickeln in der Folge selbst ein instrumentelles Verhältnis zur Welt: Verinnerlichte Rationalitäts-, Effizienz und Wettbewerbsimperative verdinglichen die Beziehungen, die das Subjekt zu anderen Personen und Objekten unterhält. ${ }^{3}$

c) Das gute oder nicht-entfremdete Leben, so Rosas normative Grundthese, ist ein resonantes Leben. Subjekt und Welt stehen sich nicht indifferent oder gar repulsiv gegenüber, sondern stehen in einem „Antwortverhältnis“ (Rosa 2017, 348). Seinen Begriff der Resonanz versteht Rosa zum einen als Erweiterung, zum anderen als Modifikation von Honneths Anerkennungstheorie. Diese sei zu erweitern, weil sie lediglich eine intersubjektive Dimension von Welterfahrung anspreche: Neben den Anerkennungsbeziehungen der Liebe, Achtung und sozialen Wertschätzung, die Honneth in Anschluss an Hegels Sittlichkeitslehre entfaltet, seien in der Moderne auch ,religiöse Erfahrungen, ästhetische Erfahrungen und Naturerfahrungen [...] von zentraler Bedeutung“ (ebd.). Obgleich das Subjekt hier keine Beziehung zu einem gleichartigen Subjekt aufbaut (horizontale Resonanzachse), sondern zu Gegenständen (diagonale Resonanzachse) oder zu etwas, was das Subjekt „umgreift““ (ebd.) (vertikale Resonanzachse), machen die Menschen in Religion, Kunst und Natur gleichwohl Resonanzerfahrungen: Sie spüren hier ,die Verbindung oder die Begegnung mit einem Anderen, das zu ihnen in einer inneren Beziehung, in einem Antwortverhältnis zu stehen scheint, das für sie eine gleichsam intrinsische Bedeutung hat, weil es sie berührt oder ergreift" (ebd.). Honneths Anerkennungstheorie müsse zudem modifiziert werden, weil sie den sozialen Bereich - ähnlich wie Pierre

\footnotetext{
3 Paradigmatisch steht hierfür der sozial isolierte und stets von Burnout bedrohte High-speed Surfer, der die (Wellen-) Bewegungen des Marktes antizipiert und sich ihnen flexibel anpasst. Als weitere Formen spätmoderner Lebensführung beschreibt Rosa den Drifter, der beim Sprung von einer Welle zur nächsten scheitere und fortan hilflos den Bewegungen des Marktes ausgeliefert ist, sowie den Fundamentalisten, der der sinnlosen Steigerungslogik durch eine religiöse oder politische Weltanschauung zu entkommen trachtet (Rosa 2011).
} 
Bourdieu - auf einen Ort des Kampfes um ein positionales Gut reduziere. Selbst wo Anerkennung wechselseitig sei, handele es sich streng genommen um zwei getrennte Akte: „Ich anerkenne Dich, Du anerkennst mich, während es Resonanz nur als dynamische Verbindung gibt. Das Ziel oder das Ergebnis dieser Verbindung ist eine wechselseitige Transformation (weshalb ich Resonanzbeziehungen als Anverwandlung von Welt beschreiben möchte), während Anerkennung zumindest die Gefahr der Reifizierung oder Fixierung des Gegebenen birgt“" (349). Während Anerkennung ein einseitig akkumulierbares und umkämpftes Gut sei, ergäbe ein Kampf um Resonanz keinen Sinn. Resonanz lasse sich nicht auf die eine oder andere Seite schlagen, sondern sei das, was Subjekt und Welt verbinde. Eben diese Verbindung sei in einem Zustand der Entfremdung gestört. Rosa teilt ,mit Rahel Jaeggi die Auffassung, dass Entfremdung sich als eine Beziehung der Beziehungslosigkeit verstehen lässt. Entfremdung bezeichnet einen Modus der Beziehung, in dem sich Subjekt und Welt innerlich unverbunden gegenüberstehen, in dem die Anverwandlung eines Weltausschnitts misslingt.“ (ebd., 350)

Ad a) Rosas Beschleunigungsdiagnose erscheint angesichts der zurückliegenden und gegenwärtigen Veränderungen in Arbeits- und Lebenswelt intuitiv plausibel. Der neoliberale Umbau westlicher Gesellschaften, die in einem rasanten Tempo voranschreitende Transformation von Ländern wie China oder Indien, die digitale Revolution, die Flexibilisierung von Arbeitsbiographien und Arbeitszeiten etc. stützen Rosas These zunächst. Als problematisch erweist sich jedoch die spezielle Annahme, dass sich in allen sozialen Bereichen Prozesse feststellen lassen, die (bei prozentual gleichbleibenden Steigerungsraten) substantiell ein exponentielles Wachstum in Gang setzen. In der modernen Politik beispielsweise müssen die um ihre Wiederwahl bangenden Parteien zwar auf soziale Entwicklungen Rücksicht nehmen, aber weder im Hinblick auf die Regierungsdauer noch im Hinblick auf die Anzahl der erlassenen Gesetze lässt sich ernsthaft von einem exponentiellen Wachstum sprechen. Schon die Terminologie, die Rosa verwendet, belegt dies: Moderne Gesetzgebung sei auf ,stetige Entwicklung, Veränderung und Anpassung angelegt" (ebd., 340). Er verwendet hier einen deutlich schwächeren Begriff dynamischer Stabilisierung, der die Stetigkeit von Anpassungsvorgängen betont, das heißt gerade keine endogene Radikalisierungstendenz impliziert und sich vom adaptiven Reproduktionsmodus nicht mehr fundamental unterscheidet. Rosa, so ließe sich sein Changieren zwischen einem starken und einem schwachen Konzept dynamischer Reproduktion deuten, trägt hier, ohne dies zu reflektieren, einer sachlichen Differenz Rechnung: Außerökonomische Handlungsbereiche mögen strukturell von der kapitalistischen Ökonomie abhängig und von ihr auch zunehmend intern affiziert sein, gehorchen aber dennoch anderen Grundprinzipien und weisen deshalb keine vergleichbare Eskalationslogik auf.

Ad b und c) Rosa möchte mit seinem Resonanzkonzept eine Theorie des Guten umreißen, ohne in ein essentialistisches Fahrwasser zu geraten. Seine Sozialkritik fußt also einerseits auf einem kulturunabhängigen inhaltlichen Kriterium Menschen streben (schon als Säuglinge) Resonanzerfahrungen an, die die gegenwärtige Konkurrenzgesellschaft systematisch untergräbt -, andererseits legt dieses inhaltliche Kriterium nicht fest, welche konkreten Resonanzbeziehungen idealerweise unterhalten werden sollten. Resonanz soll der Entfremdung oder Verdinglichung 
„wenigstens die vagen Konturen eines Positiven entgegensetzen, das offen genug ist, um der unhintergehbaren Pluralität der substantiellen ethischen Konzeptionen des Guten gerecht zu werden.“ (ebd., 353) Relativ schwache (und damit wenig angreifbare) anthropologische Voraussetzungen ${ }^{4}$ kombiniert mit Liberalität gegenüber individuellen Lebensentwürfen und anderen Kulturen, das erscheint ebenso attraktiv wie der sozialphilosophische Zuschnitt der Resonanztheorie, die ja nicht nur Selbstwirksamkeitserfahrungen mit Gegenständen sowie instrumentell motivierte interpersonelle Beziehungen umfasst, sondern auch Phänomene des kollektiven Lebens in den Blick zu nehmen vermag (selbstzweckhafte Resonanzbeziehungen in der Erwerbsarbeit beispielsweise). Gleichwohl sind diese Vorteile der Resonanztheorie mit erheblichen Nachteilen erkauft:

1. Resonanz ist ein unvollständiger und darum ergänzungsbedürftiger (oder zu ersetzender) ethischer Maßstab; Resonanz kann niemals mehr als ein Teilprinzip des gesuchten normativen Grundprinzips sein. Nehmen wir die utilitaristische Ethik, um dies zu verdeutlichen (vgl. Höffe 2008). Der Utilitarismus ist eine Moralphilosophie, die die Konsequenzen einer Handlung, Handlungsregel oder Charakterdisposition beurteilt (Folgenprinzip) und die in ihren klassischen Varianten Lustmaximierung oder Unlustminimierung als höchsten Wert angibt (hedonistisches Prinzip). Beide Teilprinzipien zusammengenommen machen den Utilitarismus allerdings noch nicht zu einer das rationale Selbstinteresse einschränkenden Ethik, weshalb es der Ergänzung durch ein weiteres Teilprinzip bedarf: Zu maximieren, sagt der Utilitarist, sei das (Gesamt-) Wohlergehen aller, die von einer Handlung oder Handlungsregel betroffen sind (Sozialprinzip). Ein solches, das wertethische Prinzip spezifizierendes Teilprinzip fehlt in Rosas Resonanztheorie. Dieser Einwand lässt sich auch dann aufrechterhalten, wenn wir davon ausgehen, dass Rosa kein Utilitarist ist, sondern stattdessen für unveräußerliche Individualrechte eintritt. Denn auch die Unveräußerlichkeit der Rechte ist begründungsbedürftig und würde von der Resonanz zu einem anderen ethischen Maßstab führen. Warum soll das Glück von Einzelnen nicht dem Gesamtwohlergehen geopfert werden dürfen? Häufig lautet die Antwort, dass die Würde des Menschen dies verbiete. Aber welche Eigenschaft verleiht dem Menschen seine Würde? In der christlich-jüdischen ebenso wie in der säkular-kantischen Tradition ist es seine Vernunftbegabung. Nur aufgrund seiner Anlage zur Freiheit hat der Mensch ,eine Würde (einen absoluten inneren Werth), wodurch er allen anderen vernünftigen Weltwesen Achtung für ihn abnöthigt, sich mit jedem anderen dieser Art messen und auf den Fuß der Gleichheit schätzen kann.“ (Kant 1797/1907, 435) Dass das Resonanzprinzip sowohl utilitaristisch als auch deontologisch ausbuchstabiert werden kann, belegt, dass es sich nur um ein unselbständiges ethisches Prinzip handeln kann.

2. Resonanz ist kein reines Mangelgut, sondern kann, ökonomisch gesprochen, auch einen negativen Grenznutzen aufweisen. Ab einem bestimmten Zeitpunkt führt mehr Resonanz nämlich nicht zu einem lebenswerteren Leben, sondern wird regel-

\footnotetext{
${ }^{4}$ Die kulturübergreifende Annahme, dass der Mensch auf Resonanz geeicht ist, findet sich in früheren Schriften (wie „Kapitalismus als Dynamisierungsspirale“) so nicht. Aussagen über die menschliche Natur lehnt Rosa hier noch rundweg als paternalistisch ab; der Kritikmodus ist rein immanent.
} 
recht zu einer Belastung, wie beispielsweise beim Pflegepersonal, das eine gewisse emotionale Distanz zu den Patienten aufbauen muss, um sich selbst psychisch zu schützen. Mehr Resonanz ist also nicht automatisch besser (vgl. Henning 2017, 70). Und deshalb ist Resonanz auch kein geeigneter Kandidat, um die wertethische Frage, was in sich gut ist oder was den höchsten Wert im Leben ausmacht, überzeugend zu beantworten.

3. Die Konturen des guten Lebens, die Rosa zeichnet, sind nicht nur vage, sondern viel $z u$ vage. Weil bereits Resonanz als solche Maßstab der Sozialkritik sein soll, kann Rosa nicht mehr zwischen moralisch wünschenswerten und moralisch abzulehnenden Resonanzen unterscheiden (vgl. Henning 2017, 71). Der Preis, den eine Theorie bezahlen muss, die aus Gründen der Liberalität vollkommen offenhalten will, wie, womit, mit wem und zu welchen Zwecken die Menschen Resonanzbeziehungen eingehen - wichtig ist nur, dass sie welche eingehen -, ist normative Beliebigkeit und Verlust utopischer Orientierung.

\section{Kants Freiheitslehre}

Die Geschichte des Sozialismus, daran hat Axel Honneth (2015) eindrucksvoll erinnert, war immer auch eine Geschichte normativ angeleiteter Kritik und normativer Erwartungen an eine bessere Gesellschaft. Selbst zu Zeiten der zweiten Internationale waren Objektivismus und Moralskepsis nicht allein am Platz; so waren insbesondere Vertreter des Ethischen Sozialismus (wie Hermann Cohen oder Karl Vorländer) bemüht, der Kritik am Kapitalismus durch einen Rückgriff auf die kantische Moralphilosophie einen sicheren Halt zu geben. Diese Tradition wirkt bis heute fort (u. a. bei Kuhne 2017, 2015; Zunke 2011; Ruschig 2010). Frank Kuhne beispielsweise ist der Ansicht, dass Marx zwar noch in seinem Spätwerk an einem reduktionistischen Basis-Überbau-Schema und einer materialen Geschichtsphilosophie verbal festgehalten habe, in seiner Analyse und Kritik des Kapitalismus jedoch implizit auf die Selbstzweckformel des kategorischen Imperativs zurückgreife. Der Skandal sei auch für Marx, dass das Kapital als ,,automatisches Subjekt“ (Marx 2001, 169) die Menschen zu bloßen Mitteln der Selbstverwertung degradiere. Wie ich im Folgenden zeige, ist diese interessante Option, die verselbständigten ökonomischen Strukturen im Lichte der Selbstzwecklichkeit des Menschen zu beurteilen, jedoch mit einigen Problemen verbunden, die mit der kantischen Ethik und Freiheitslehre zusammenhängen.

Wie bereits die intensive Diskussion der Zeitgenossen von Immanuel Kant zeigt, lassen sich in dessen moralphilosophischen Schriften mindestens zwei verschiedene Freiheitsbegriffe identifizieren. Der erste Freiheitsbegriff lässt sich der Grundlegung zur Metaphysik der Sitten von 1785 und der zweiten Kritik von 1788 entnehmen. Beiden Schriften zufolge ist ein Wille genau dann frei zu nennen, wenn er nicht durch sinnliche Beweggründe, sondern allein durch das von der reinen praktischen Vernunft gewirkte moralische Gesetz bestimmt ist. Anders gesagt: Freiheit wird negativ als Unabhängigkeit des Willens von sinnlichen Antrieben der Lustgewinnung oder Unlustvermeidung definiert, denn jedes Handlungsprinzip, das auf einen Lust- oder 
Unlustgefühle hervorrufenden Gegenstand zielt, ist Kant zufolge nur ein „Princip der Selbstliebe oder eigenen Glückseligkeit“ (Kant 1788/1913, 22). Positiv formuliert besteht Freiheit in der Selbstgesetzgebung der Vernunft, das heißt, sie wird mit der Nötigung identifiziert, die von einer Seite des innerlich zerrissenen Menschen ausgeht: Überwiegt der Vernunftzwang die Naturzwänge, so neigt sich das Zünglein der Waage zur Freiheit. Das bereits von Johann Heinrich Abicht gebrauchte Bild der „Willenswaage“ (Abicht 1789/1975, 230) ist insofern aufschlussreich, als es die deterministischen Implikationen verdeutlicht, die Carl Christian Erhard Schmid seinerzeit zum Intelligiblen Fatalismus ausbaute: Ob eine Maxime oder Handlung moralisch ausfällt oder nicht, ist abhängig vom Gewicht der sinnlichen Hindernisse einerseits und vom Gewicht der praktischen Vernunft andererseits, das heißt Resultat der Wirkung zweier entgegengesetzter Determinationsgrößen. Damit, so schon Leonhard Creuzer, sind Handlungen jedoch nicht länger zurechenbar, denn ohne einen Akt der Entscheidung sind weder unmoralische Maximen und Handlungen individuell verschuldet, noch verdienen moralische Maximen und Handlungen ein Lob: „Ich kann alsdenn nicht mehr sagen: Ich soll moralisch handeln, sondern ich ти $\beta$ moralisch handeln. Der Begriff von Verdienst verliert also eben so gut seine Gültigkeit, als der Begriff von Schuld.“ (Creuzer 1793/1975, 287) Die Idee, den guten Willen in einer selbsttätigen, aber dennoch unverfügbaren Vernunft zu verankern, hat sich damit als ein untauglicher Versuch erwiesen, den Sinn des Wortes Freiheit zu explizieren. Wer nicht anders kann, als dem Diktat der Vernunft zu folgen, ist nicht frei, sondern ohnmächtig.

Noch bevor Kant sich selbst daransetzte, den Mangel zu beheben, der seine Kritik der praktischen Vernunft auf eine Spielart des Determinismus zusteuern ließ, interpretierte Carl Leonhard Reinhold die kantischen Schriften auf eine instruktive, wenngleich nicht weniger problematische Art und Weise. Der Begriff des freien Willens sei nur dann zu retten, wenn er „das Vermögen der Person“ bezeichne, ,sich selbst zur Befriedigung oder Nichtbefriedigung eines Begehrens entweder nach dem praktischen Gesetze oder gegen dasselbe zu bestimmen“ (Reinhold 1792/1975, 255). Die Freiheit des Menschen besteht demnach nicht nur darin, ein kurzfristiges Begehren zugunsten eines langfristigen Begehrens zurückzustellen (Langfristlustkalkulation) oder die Forderungen der praktischen Vernunft den Forderungen der Sinnlichkeit vorzuziehen, sondern sie umfasse darüber hinaus das Vermögen, auch den Imperativen der praktischen Vernunft eine Absage zu erteilen und stattdessen das sinnliche Begehrungsvermögen bei der Bestimmung des Willens vorzuziehen. Reinhold ist sich der dezisionistischen Natur seiner These durchaus bewusst: „Die freie Handlung ist darum nichts weniger als grundlos.“ „Fragen: Warum der Wille sich auf diese oder jene Art bestimmt habe, heißt fragen: Warum er frei ist? Voraussetzen, er bedürfe eines von ihm selbst verschiedenen Grundes, heißt ihm seine Freiheit absprechen." (Reinhold 1792/1975, 261, 262) Ganz ähnlich argumentiert Kant, der mit Reinholds Schriften vertraut war, wenig später in Die Religion innerhalb der Grenzen der bloßen Vernunft im Hinblick auf die Bösartigkeit. Der Mensch sei zwar kein „teuflische[s] Wesen“, welches den Verstoß gegen das moralisch Gesollte zur Norm erhebt - der Mensch verstößt nicht „rebellischerweise“ (Kant 1794/1907, 36) gegen das Sittengesetz. Jedoch lasse schon ein kurzer Blick in die menschliche Geschichte keinen anderen Schluss zu, als dass es einen „,natürliche[n] Hang des Menschen 
zum Bösen“ (ebd., 29) gebe. Unter dem Bösen versteht Kant eine aus freien Stücken erfolgte Inversion der Triebfederhierarchie. Wer böse ist, macht die Befolgung des moralischen Gesetzes von der Erfüllung sinnlicher Ansprüche abhängig, das heißt er ordnet die Sittlichkeit der Sinnlichkeit unter - eine Entscheidung, die ,am Ende doch in einer freien Willkür gesucht werden muß, mithin zugerechnet werden kann“ (ebd., 37). Diesen ursprünglichen Wahlakt, der kausal nicht weiter zurückgeführt werden kann, sondern uns als ,absolute Spontaneität der Willkür“ (ebd., 24) auf ewig unerklärlich, ,unerforschlich“ (ebd., 43) bleibt, hat Leonhard Creuzer als vernunftwidrig zurückgewiesen: „Ist aber wohl eine Freiheit vernünftig denkbar, die ein und dasselbe Wesen gleichvermögend macht für kontradiktorisch entgegengesetzte Handlungen?" (Creuzer 1793/1975, 275) Wenn Freiheit darin bestehen soll, in ein und derselben Situation zwischen einer Handlung und ihrem direkten Gegenteil wählen zu können, dann läuft dies auf pure Dezision und damit auf „Abhängigkeit vom absoluten Zufall"“ (ebd.) hinaus. So verstanden wäre Freiheit, wie schon Abicht bemerkt, eine „Wahl ohne Grund, oder welches nun einerlei ist: Nichts.“ (Abicht 1789/1975, 230) Warum? Das entscheidende Argument liefert Ansgar Beckermann: „Die Gründe, angesichts deren eine Person entscheidet, gehören mit zu der Situation, in der sie sich entscheidet. Wenn sie sich für $A$ entscheidet, entscheidet sie sich angesichts dieser Gründe für $A$. Und wenn sie sich für $B$ entscheidet, entscheidet sie sich angesichts genau derselben Gründe für $B$. Wenn jemand angesichts derselben Gründe einmal die Alternative $A$ und das andere Mal die Alternative $B$ wählt, ist diese Wahl selbst aber offenbar unbegründet.“ (Beckermann 2015, 134) Letztlich erweisen sich beide Stränge des kantischen Freiheitsdenkens in ihrer Konsequenz als freiheitswidrig und damit inkonsistent. Dem ersten Strang fehlt eine Ich-Instanz, die nicht unter der Gewalt der beiden widerstreitenden Triebfedern steht und der darum eine gute oder böse Maxime oder Handlung allererst zugerechnet werden kann (denn Verantwortlichkeit setzt Akteurskausalität voraus), während im zweiten, dezisionistischen Strang eine indeterminierte, von Vernunft und Sinnlichkeit gleichermaßen unabhängige Instanz eine irrationale, weil durch nichts begründete Wahl trifft (vgl. Klar 2007, 73-84).

Unterstellen wir jedoch für einen Moment, dass Kants Freiheitslehre nicht aporetisch ist ${ }^{5}$, sondern uns schlüssig darlegt, warum dem Menschen eine unbedingte Würde zukommt, die dem nur auf sich und seine Verwertung gerichteten Kapitalprozess vollkommen äußerlich ist. Was folgte daraus sozialphilosophisch? Welches Bild einer postkapitalistischen Gesellschaft vermittelt sie uns? Sehen wir von den Differenzen zwischen den oben angeführten Strängen der kantischen Freiheitslehre ab, so wird deutlich, dass beiden ein strenger Triebfederdualismus zugrunde liegt: auf der einen Seite die reine, das Bewusstsein des Sittengesetzes in uns hervorrufen-

\footnotetext{
5 Auch bei der Erörterung des hegelschen Freiheitsbegriffs werde ich voraussetzen müssen, dass das klassische Problem der Vereinbarkeit von Freiheit und Determination bei Hegel bereits gelöst ist oder ohne gravierende konzeptionelle Änderungen von ihm hätte gelöst werden können. Er selbst schenkte dem Problem jedenfalls keine große Aufmerksamkeit - möglicherweise, weil er „Kausalerklärungen für weniger grundlegend als Finalerklärungen“ hielt (Quante 2011, 27), möglicherweise, weil er den Wahl- und Willkür-basierten Zuschnitt der damaligen Willensfreiheitsdebatte ablehnte. Gleichwohl ist die Frage berechtigt, ob Hegel zu einer kompatibilistischen oder inkompatibilistischen Position neigte. Zu den wenigen Forschern, die sich an diese schwierige Frage heranwagen, gehören Pippin (2005) und Knappik (2013, 356 ff.).
} 
de praktische Vernunft (die gemäß der ersten Freiheitslehre auch den empirischen Willen hinreichend zu bestimmen vermag), auf der anderen Seite die sinnlich-heteronomen, ausschließlich dem Prinzip der Selbstliebe verpflichteten Antriebe. ${ }^{6}$ Eine Handlung erfolgt deshalb entweder rein aus Pflicht und ist damit moralisch - in diesem Fall waren für die Bestimmung des Willens überhaupt keine sinnlichen Antriebe maßgeblich -, oder die Handlung zielt auf Gegenstände, die Lust oder Unlust im Individuum hervorrufen, und ist somit amoralisch (jedoch nicht zwingend unmoralisch). An Kants Metaphysik der Sitten lässt sich demonstrieren, welche sozialphilosophischen Auswirkungen es hat, wenn man in dieser Schere von universeller reiner Vernunft und partikularen hedonistischen Trieben denkt. Soziale Institutionen lassen sich nur noch daraufhin befragen, ob sie das äußere menschliche Zusammenleben zumindest so organisieren, dass es den Forderungen der Vernunft entspricht, selbst wenn sich die Menschen subjektiv gar nicht für die Forderungen der Vernunft empfänglich zeigen, sondern vielmehr ihre Naturseite zur Richtschnur des Handelns erheben. Der Rechtsstaat muss dann qua Zwang sicherstellen, dass die dem Prinzip der Selbstliebe folgenden Partikularwillen nicht die Grenzen ihrer legitimen Freiheitsausübung überschreiten und somit wenigstens der moralischen Pflicht gemä $\beta$ handeln (ohne durch sie motiviert zu sein). Anders als in der von Hobbes begründeten Tradition der Vertragstheorie geht der Staat bei Kant zwar nicht aus den Klugheitsüberlegungen rationaler Egoisten hervor. Das Recht als ,Inbegriff der Bedingungen, unter denen die Willkür des einen mit der Willkür des andern nach einem allgemeinen Gesetze der Freiheit zusammen vereinigt werden kann“ (Kant 1797/1907, 230), ist offensichtlich eine auf äußere Handlungen spezifizierte Variante des kategorischen Imperativs und somit ein genuines Vernunftrecht. Den klassischen staatsphilosophischen Kontraktualismus und die kantische Moral-/ Rechtsphilosophie trennen Welten. Einerseits. Andererseits entsteht diese radikale Differenz lediglich durch die eine Seite des kantischen Triebfederdualismus: durch die die Würde des Menschen ausmachende reine Vernunft. Die andere Seite des Triebfederdualismus - unteres Begehrungsvermögen, Selbstliebe, Streben nach Lust, Vermeidung von Unlust, selbstsüchtige Neigungen, Wetteifer, unaufhebbarer (und darum die Notwendigkeit des Privateigentums an Produktionsmitteln begründender) Willensantagonismus - entspricht jedoch ziemlich genau dem theoretischen Fundament, auf dem Hobbes seinen Leviathan aufbaute. Es ist daher auch kein Zufall, dass Kant die Institution der Ehe auf einen Vertrag zum wechselseitigen Gebrauch der Geschlechtseigenschaften reduziert (Kant 1797/1907, 277f.) und zuweilen auch für den Staat kontraktualistische Begründungen heranzieht: „Das Problem der Staatserrichtung ist, so hart es auch klingt, selbst für ein Volk von Teufeln (wenn sie nur Verstand haben) auflösbar“ (Kant 1795/1923, 366). Wer Sinnlichkeit nur als das heteronome Andere der reinen Vernunft denkt, wird keinen Begriff sittlicher sozialer Institutionen oder kommunaler Freiheit entwickeln können.

\footnotetext{
6 ,Alle materiale praktische Principien sind, als solche, insgesamt von einer und derselben Art, und gehören unter das allgemeine Princip der Selbstliebe oder eigenen Glückseligkeit.“ (Kant 1788/1913, 22).
} 


\section{Hegels Freiheitslehre}

Die immanenten Probleme der kantischen Moralphilosophie haben schon in der ersten Generation der Kritischen Theorie dazu geführt, sich intensiv mit Hegels Rechtsphilosophie auseinanderzusetzen. Sie war keineswegs nur (herrschaftslegitimierender) Stein des Anstoßes, sondern galt in sachlicher, methodischer und normativer Hinsicht als eine der größten und produktivsten Herausforderungen. Aktuell steht für diese Rezeptionslinie insbesondere der Name Axel Honneth. Studien wie Leiden an Unbestimmtheit (2001) und Das Recht der Freiheit (2011) sind von der hegelschen Grundintention getragen, den Begriff des freien Willens in einer Art und Weise zu verstehen, die es erlaubt, mit Kant (und seinem Vernunftanspruch) über Kant hinauszugehen. Weil ich diese Intention teile, werde ich im Folgenden zunächst die Grundzüge der hegelschen Freiheitslehre darstellen, bevor ich der Frage nachgehe, worin genau ihr kritisches Potential liegt.

In der Einleitung seiner Rechtsphilosophie unterbreitet Hegel den wegweisenden Vorschlag, den freien Willen als Einheit von zwei entgegengesetzten Momenten zu begreifen. Das erste der beiden Momente bezeichnet Hegel als ,reine Unbestimmtheit“ (Hegel 2013, 32) oder als „Wille überhaupt“ (Hegel 2005, 43): ,Ich will“. Wie bereits die Zusammensetzung aus Subjekt und Prädikat andeutet, impliziert der Wille überhaupt zweierlei. Er beinhaltet zum einen die Vorstellung eines abstrakten Ichs, denn nur wer in der Lage ist, gedanklich von allen Attributen abzusehen, die ihn zu einer konkreten Person machen - Biografie, lebensweltliche Bezüge, körperliche und geistige Konstitution, Wünsche und Begierden -, könne nach Hegel auch frei genannt werden. Der Wille überhaupt beinhaltet zum anderen die Absicht einer Zweckrealisation (,Ich will'). Gemeint ist damit nicht etwa die Absicht, einen spezifischen Zweck zu realisieren, sondern es geht erst einmal nur um die begriffliche Implikation, dass sich ein Wille überhaupt ohne eine Zweckrealisationsabsicht überhaupt nicht denken lässt. Damit gerät der Wille überhaupt jedoch in ein Dilemma. Er ist zwar einerseits das sich ins Praktische übersetzen wollende oder sich Wirklichkeit geben wollende Denken, andererseits ist unklar, wie es sich auf keinen konkreten äußeren Gegenstand festlegen können soll - nicht nur, weil jede über das abstrakte Wollen hinausgehende Prädikation des Ichs seiner Uneingeschränktheit unweigerlich Abbruch täte, sondern auch, weil mit dem Abstraktionsvorgang, der das sich nur auf sich beziehende Ich erst hervorbrachte, auch die inhaltlichen Kriterien verloren gingen, die für eine Bestimmung des Willens erforderlich wären. Ein Freiheitverständnis, das derart am Prinzip des Wollens orientiert ist, mündet entweder in blanker Willkür, bei der aus einer begrenzten Anzahl vorgefundener Optionen eine unbegründete Wahl getroffen wird, oder es resultiert in dem schieren Unvermögen, sich überhaupt noch auf Anderes (Dinge, Personen, Institutionen) einzulassen und zweckbezogene Entscheidungen zu treffen. In beiden Fällen scheitert der Versuch, die Freiheit des Willens durch einen reinen Selbstbezug sicherzustellen.

Das Distanzierungsvermögen des Verstandes kann also nicht das letzte Wort in der Freiheitsdebatte sein. Der Selbstbezug muss durch Fremdbezüge vermittelt sein, wenn er nicht in Unfreiheit umschlagen soll. Hegel drückt diesen Sachverhalt terminologisch so aus, dass die reine Unbestimmtheit der Ergänzung durch das Moment der „Bestimmtheit“ (Hegel 2013, 33) bedarf: ,Ich will den Gegenstand X“. Die 
Festlegung auf einen besonderen Zweck, die aus der Perspektive der reinen Unbestimmtheit als Einschränkung der Autonomie des Ichs erschien, stellt Hegel zufolge auch eine Form der Befreiung dar: eine Befreiung vom selbstauferlegten Reinheitszwang, konkreten Zwecken zu entsagen. Während also die Allgemeinheit einer bloß negativen Freiheit, die sich von den Gegenständen krampfhaft abschottet, selbst zu einem Partikularen degradiert und leerläuft, ermöglicht der Gegenstandsbezug eine positive, das heißt uns zu etwas befähigende Freiheit. Erst die Selbstbeschränkung erzeugt neue Spielräume des Denkens und Handelns. Andererseits mahnt uns Hegel, die inhaltliche Seite des Willens genauso wenig absolut zu setzen wie ihre abstrahierende Verstandesseite, denn dann liefe der Wille auf bloße Determination durch Gegebenes (Begierden, Triebe) hinaus.

Wie eingangs erwähnt, sieht Hegel die Lösung des Problems darin, den freien Willen als Einheit der beiden einseitigen Momente zu denken. Wie ist das zu verstehen? Schließen sich Allgemeines (Ich) und Besonderes (etwas) zu einem konkretAllgemeinen zusammen (in Hegels Terminologie: zur Einzelheit), dann gelingt es dem Ich, sich mit einer konkreten Willensbestimmung zu identifizieren; der Inhalt ist nicht länger ein Fremdes, sondern er wird zu seinem Inhalt. Erst durch diesen Aneignungsakt gelingt es dem Ich, die Freiheitsmomente der beiden Seiten des Willens zu wahren und ihre selbstdestruktiven Tendenzen zu neutralisieren.

Auf den ersten Blick erinnert dies stark an Harry Frankfurts hierarchisches Motivationsmodell, in dessen Mittelpunkt die Aneignung oder Nicht-Aneignung von Wünschen steht. Anders als Tiere hätten Menschen nicht einfach nur Wünsche, sondern sie können sich in reflexiv-evaluierender Weise auf diese Wünsche beziehen (vgl. Frankfurt 1971, 6). Für Personen ist es charakteristisch, dass sie sich die Frage stellen können, ob ein gegebener Wunsch selbst wünschenswert ist und daher handlungswirksam werden sollte. Frankfurt veranschaulicht dies am Drogenabhängigen wider Willen. Dieser wünscht sich vergeblich, den handlungswirksamen Wunsch, Drogen zu nehmen, nicht zu haben. Sein Wille ist nicht frei, weil er nicht in der Lage ist, den Wunsch erster Stufe (Drogen zu nehmen) der Volition zweiter Stufe (keine Drogen zu nehmen) anzupassen. Sein effektives Wollen wird von ihm daher als etwas Fremdes empfunden, als etwas, womit er sich nicht identifizieren kann (vgl. Frankfurt 1971, 12). Ganz ähnlich argumentiert Hegel im Hinblick auf innere Zwänge: „Zorn, Leidenschaft ist auch das Meinige, aber man sagt doch, ich bin außer mir. Formell bin ich da wohl Wille, aber nur formell. Ich kann einen Inhalt wohl zu dem meinigen haben, aber so, daß er von einer Seite nicht der meine ist." (Hegel 2005, 45, Herv. von mir)

Auf den zweiten Blick zeigen sich bedeutende Unterschiede zwischen Frankfurts und Hegels Willenskonzeption - wobei nicht jede Abgrenzung, die in der Literatur vorgenommen wird, auch vollends überzeugt. So ist kritisch angemerkt worden (u. a. von Guckes 2001, 14), dass bei Frankfurt die Harmonie von Wille erster Stufe und Volition zweiter Stufe hinreichende Bedingung für die Existenz von Willensfreiheit sei; sobald sich ein Subjekt mit seinen effektiven Wünschen identifiziere, sei sein Wille frei. Es gibt einige Stellen, die eine solche Deutung zulassen. Wohlwollend interpretiert ist die Identifikation jedoch auch bei Frankfurt nur eine notwendige Bedingung. Er verdeutlicht dies am Beispiel eines Drogenabhängigen, der von seiner Sucht nicht nur nicht loskommen kann, sondern der es auch gar nicht will: Wunsch 
erster Stufe und Volition zweiter Stufe entsprechen einander, und dennoch verfügt der Süchtige nicht über Willensfreiheit, weil er nicht über die Fähigkeit verfügt, den gegenteiligen Wunsch (keine Drogen zu nehmen) handlungswirksam werden zu lassen, wenn er ihn denn nur hätte (vgl. Frankfurt 1971, 19). Willensfreiheit meint bei Frankfurt nicht nur die Kongruenz von Wunschhierarchien, sondern setzt darüber hinaus das Vermögen voraus, den effektiven Wunsch erster Stufe durch eine Volition zweiter Stufe steuern zu können.

Worin unterscheiden sich beide Willensmodelle dann? Rufen wir uns in Erinnerung, dass Hegel den freien Willen als Einheit des Moments der reinen Unbestimmtheit und des Moments der Bestimmtheit definiert. Frankfurt versteht unter Willensfreiheit die ein Steuerungsvermögen voraussetzende Einheit von Volition zweiter Stufe und Wunsch erster Stufe. Beide Begriffspaare lassen sich insofern nicht aufeinander abbilden, als nicht nur das Rohmaterial unserer Wünsche (Stufe 1), sondern auch die höherstufigen Wünsche unter Hegels Begriff der Bestimmtheit fallen: Sowohl Quelle als auch Qualität der Volitionen zweiter Stufe sind für Frankfurt ausdrücklich unerheblich für die Frage der Willensfreiheit. Sie können genauso launenhaft und unmoralisch sein wie die vorgängigen Wünsche, auf die sie sich beziehen (vgl. Frankfurt 1971, 13). Anders gesagt, bei Frankfurt gibt es kein Pendant zum qualitativen Korrektiv der reinen Unbestimmtheit.

Dass für Hegel der Wille nur dann frei zu nennen ist, wenn er vermittelt über einen Fremdbezug seine innere Allgemeinheit (Unabhängigkeit) geltend macht und darum noch in seiner inhaltlichen Bestimmtheit ganz bei sich bleibt, kommt in der berühmten Sentenz zum Ausdruck, dass der freie Wille ,den freien Willen will.“ (Hegel 2013, 48) Der trotz aller Kritik von Kant übernommene Grundgedanke lautet, dass der freie Wille reflexiv in dem Sinne ist, dass er die Freiheit selbst zu seinem Inhalt hat. Die hegelsche Rechtsphilosophie ist ihrer Grundintention nach nichts anderes als der großangelegte Versuch, die verschiedenen Entwicklungsstufen der sich selbst wollenden Freiheit darzustellen. Dass der freie Wille sich selbst zum Endzweck hat und damit über die Partikularität der natürlichen Triebe erhebt, verdeutlicht Hegel noch in der Einleitung durch eine Kritik des Eudämonismus. Häufig sei die menschliche Freiheit in der Fähigkeit gesehen worden, ein kurzfristiges Begehren zugunsten einer Vorstellung von Glückseligkeit zurückzustellen oder gar abzuweisen. Hegel ist weit davon entfernt, diese Freiheit geringzuschätzen. „Diese Stufe ist von hohem Wert. Es ist ein allgemeines Prinzip, worunter das Besondere subsumiert. Das Recht der Leidenschaft ist geliehen, indem sie nicht allein für sich das Richtige ist, sondern sie muss verglichen werden mit einem Zweck und ist so einer Regel unterworfen." Der Mangel eudämonistischer Lehren besteht nach Hegel allerdings darin, dass ihr Inhalt (Glückseligkeit) den natürlichen Trieben entlehnt ist und nur ,die Form der Allgemeinheit haben soll“. „,Die Allgemeinheit der Glückseligkeit nimmt ihren Inhalt aus dem Stoff der Triebe, bestimmt sich nicht selbst. Dies ist das Unfreie dieses Endzwecks.“ (Hegel 2005, 53) Mit anderen Worten: Während die Langfristlustkalkulation noch im Dienste der vorgängigen Triebe steht, erfordert Willensfreiheit eine Sublimierung der natürlichen Triebe zu sittlicher Motivation. Damit die beiden Momente des Willens sich nicht mehr als fremde gegenüberstehen, müssen Bildungsprozesse in Gang gesetzt werden, die die äußere Natur, die innere Natur sowie die zweite Natur der sozialen Institutionen betreffen. 
Im ersten Teil der Rechtsphilosophie - überschrieben mit „Das abstrakte Recht“ - entwickelt Hegel ausgehend vom Freiheitsbewusstsein der reinen Unbestimmtheit die Grundbegriffe des Privat- und Strafrechts. Die Fähigkeit des Menschen, von seiner konkreten Ich-Identität und Lebenswelt zu abstrahieren, das heißt einen qualitätslosen Begriff des Ichs zu bilden, durch den er sich als einzelner und zugleich als identisch mit allen anderen weiß, findet seinen institutionellen Ausdruck im egalitären Recht. Vor ihm gilt man nicht länger als Vertreter eines besonderen Standes, sondern als Person, das heißt als gleicher Träger juridischer Rechte und Pflichten. Das Moment der Bestimmtheit wiederum zeigt sich in der Aneignung, insbesondere in der Bearbeitung der äußeren Natur: Indem der Mensch seinen Willen in eine äußere Sache legt, erfährt er sich erst wirklich als freies Wesen. Er modelliert die Natur, vergegenständlicht sein Inneres, formt die Welt der Sachen gemäß den eigenen Zwecken (welche immer das auch seien). ,[I]ch gebe dem Lebendigen als meinem Eigentum eine andere Seele, als es hatte; ich gebe ihm meine Seele." (Hegel 1970, 107) Der Assimilationsprozess bewirkt zum einen, dass die Dinge für die Person ihre Fremdheit verlieren (sie gehören nun zu ihm, weil sie seine Signatur tragen). Zum anderen wird allen anderen Personen durch den Akt oder das Resultat der Formierung angezeigt, dass hier bereits ein zu respektierendes Willensverhältnis vorliegt, welches den exklusiven Besitz an der Sache begründet und durch staatlichen Zwang geschützt ist. Im Zentrum des abstrakten Rechts steht das Bewusstsein negativer Freiheit. Es ist privatistisch verfasst, verlangt es doch lediglich, dass die im Personenbegriff implizierten Rechte nicht verletzt werden. Seine Eigentümlichkeit besteht folglich darin, dass es nicht das konkrete Subjekt mit seinen konkreten Bedürfnissen, Motiven und Bestimmungsgründen des Willens zum Gegenstand hat. ${ }^{7}$ Wie schon bei Kant koordiniert das (abstrakte) Recht lediglich äußere Handlungen. Es legt die vertraglich interagierenden Privatpersonen nicht auf bestimmte Zwecke fest und fordert von ihnen auch keine besondere innere Einstellung (z. B. Empathie). Vielmehr ermöglicht es moralische Gleichgültigkeit: „Hat jemand kein Interesse als sein formelles Recht, so kann dieses reiner Eigensinn sein, wie es einem beschränkten Herzen und Gemüte oft zukommt; denn der rohe Mensch versteift sich am meisten auf sein Recht.“ (Hegel 1970, 96) Diese Einstellung reflektiert nur die Einseitigkeit des formellen Rechts: Es ist unempfindlich für das, was das Individuum bewegt oder bewegen sollte.

Anders in der sogenannten „Moralität“. Diese Überschrift des zweiten Teils der Rechtsphilosophie ist durchaus irreführend. Er thematisiert am Ende zwar auch die Moral im engeren Sinne, sie ist jedoch eingebettet in eine allgemeine Handlungstheorie (siehe hierzu Quante 1993); es geht im Moralitätsabschnitt nicht nur um Ethik, sondern, im Unterschied zum abstrakten Recht, um die subjektive Seite des Willens. ${ }^{8}$ Analog zur in der Einleitung vorgenommenen Differenzierung des Willens in Wille überhaupt und bestimmter Wille unterscheidet Hegel die Handlung in Handlung überhaupt und bestimmte Handlung. Eine Handlung im Allgemeinen zeichnet

\footnotetext{
7 „Im formellen Rechte kommt es [...] nicht auf das besondere Interesse, meinen Nutzen oder mein Wohl an - eben so wenig auf [...] die Einsicht und Absicht.“ (Hegel 2013, 56).

8 „Moralisch ist hier im allgemeinen Sinn genommen, nicht als das dem Unmoralischen Gegenüberstehende." (Hegel 1983a, 91).
} 
sich dadurch aus, dass ihr ein Vorsatz zugrunde liegt. Beispielsweise trage Schuld an einer Handlung, wer diese gewollt hat - und auch die Folgen einer Handlung seien einem Subjekt zuzurechnen, wenn sie ,nichts sind als die Entwicklung der Handlung selbst" (Hegel 1983a, 94). Real gibt es solche Handlungen überhaupt freilich nicht; Handlungen sind immer schon bestimmte Handlungen, mit denen das Subjekt eine spezifische Absicht - ein besonderes Interesse - verfolgt. Es geht dabei nicht einfach partikularen natürlichen Leidenschaften nach, sondern befragt diese zuerst darauf, ob sie seinem Wohl zuträglich oder abträglich sind. Absichten sind also gedanklich vermittelte Zwecke eines Subjekts, das sich um sein individuelles Wohl sorgt. Sie zu verfolgen verteidigt Hegel leidenschaftlich gegen „mönchische Tugenden“ der Bedürfnishemmung und Aufopferung; das Prinzip der Besonderheit sei das Signum der Moderne ,und macht das Prinzip der höheren Qualität gegen die ältere Zeit aus." (Hegel 1983a, 98) Das Verlangen nach individuellem Wohl und Authentizität kann jedoch mit dem bestehenden Recht kollidieren. Grundsätzlich ist dieser Konflikt zugunsten des Rechts aufzulösen - ,und ich darf [...] mein Wohl durchaus nicht befördern und behaupten auf Kosten des Rechts“ -, im Extremfall, das heißt, wenn das Leben oder die Lebensgrundlagen einer Person gefährdet sind, greife jedoch das Notrecht. So wird einem verschuldeten Handwerker ,,sein Handwerkszeug, einem Bauer sein Ackergerät gelassen.“ (Hegel 1983a, 100) Das Wohl kann also seine Grenze am Recht finden und das Recht seine Grenze am Wohl. Aufgelöst wird dieser Widerspruch zwischen dem abstrakt-allgemeinen Willen und dem besondere Willen in der Idee des Guten. Wer das Gute will, möchte nicht nur sein besonderes Wohl, sondern will ein ,allgemeines Wohl“ (Hegel 2013, 128) befördert sehen. Solange wir uns auf dem Standpunkt der Moralität, das heißt auf dem Standpunkt des reflektierenden besonderen Willens befinden, lässt sich Hegel zufolge jedoch nicht angeben, was dieses Gute inhaltlich auszeichnet. Ein Musterbeispiel hierfür sei der kategorische Imperativ Kants, der als rein formales Verallgemeinerungsgebot zu keinen inhaltlich gehaltvollen Pflichten führe. Universalisiert gedacht mag eine den Diebstahl zulassende Maxime zwar mit dem Eigentum ihre Anwendungsbedingung untergraben und so in einen Selbstwiderspruch geraten, jedoch könne dies nur für denjenigen ein Problem darstellen, der das Eigentum bereits als schützenswerte Institution voraussetze - was in einem formalen Prüfverfahren wie dem kategorischen Imperativ einem uneingestandenen normativen Dogmatismus gleichkäme (vgl. Hegel 2013, 133). Ganz ähnlich kritisiert Hegel die Subjektivität des Gewissens. Auf der einen Seite markiere das Gewissen den entscheidenden Fortschritt im Bewusstsein der Freiheit gegenüber der antiken Sittlichkeit, auf der anderen Seite liege in der Abweisung traditioneller, religiöser, kultureller oder positiv-rechtlicher Normvorgaben eine nicht zu unterschätzende Gefahr für das soziale Zusammenleben. Weil das bloß subjektive Gewissen das Gute rein aus sich bestimmen muss, tendiert es zur pluralitätsfeindlichen wie eitlen Anmaßung, andere Subjektivität nur nach eigenen Maßstäben anzuerkennen. Wie schon der Abschnitt zum abstrakten Recht mündet auch der zur Moralität in einem pathologischen Weltverhältnis, das sich aus der Verabsolutierung eines unhintergehbaren Freiheitsmoments der Moderne ergibt.

Fassen wir noch einmal zusammen. Das formale Recht und die Moralität können insofern als Sphären des freien Willens gelten, als sie seine allgemeine und seine besondere Seite zusammenschließen. Sie tun dies auf unterschiedliche Weise. 
Charakteristisch für das abstrakte Recht ist das objektive Dasein interpersoneller Anerkennung. Unabhängig von subjektiver Zustimmung oder Ablehnung koordiniert es als Ensemble zwangsbewehrter Institutionen das äußere Handeln von Rechtspersonen, damit diese ihre individuellen Zwecke gegenstandsvermittelt verfolgen, sich dabei als selbstwirksam erfahren und schließlich als anerkannt wissen können. Anders verhält es sich in der Moralität. Hier werden nicht Individuen von einem äußeren Institutionenkomplex als formal gleiche Rechtsträger adressiert, sondern es steht das besondere, sich selbst und seinem Gewissen gegenüber verpflichtete Subjekt im Zentrum. Es bewertet nicht nur seine Zwecke im Sinne einer hedonistischen Langfristlustkalkulation, sondern fühlt sich darüber hinaus der Förderung des Allgemeinwohls verpflichtet - eines Allgemeinwohls jedoch, welches das bloß subjektive Gewissen nur willkürlich festzulegen vermag. Ein freies Gemeinwesen bedarf deshalb weiterer Freiheitssphären, die die Vorzüge des formalen Rechts und der Moralität zusammenbringen. Einerseits müssen sie ein festes Dasein im Hier und Jetzt haben, so wie es bei der objektiven Anerkennung des abstrakten Rechts der Fall ist. Sie dürfen das Gute nicht (wie in moralischen Eschatologien) in ein Jenseits verlagern, sondern müssen es institutionell stabilisieren. Andererseits dürfen sie dem Subjekt nicht als formale Koordinationsmechanismen äußerer Willkürhandlungen gegenübertreten. Damit das Subjekt bereit ist, sich für sie einzusetzen, muss es sich in ihnen wiedererkennen und mit ihnen emotional verbunden fühlen können - und dafür bedürfen sie eines konkreten inhaltlichen Profils oder eines lebensweltlichen Zuschnitts, der das Handlungs- und Erfahrungsspektrum der Individuen begrenzt und eben dadurch erweitert. Kurz: Es bedarf der Sittlichkeit. Sittlich sind nach Hegel solche Institutionen zu nennen, die nicht bloße Arrangements zur Mehrung des individuellen Vorteils darstellen (wie in den klassischen Vertragstheorien), sondern in denen das gemeinsame Leben selbst im Mittelpunkt steht. Als eine Art Erweiterung des Ichs verschwimmt in ihnen die Differenz zwischen Egoismus und Altruismus. Die Individuen setzen sich für das konkret-Allgemeine ein, weil es einen Teil ihrer Identität ausmacht. Freiheit ist damit ein komplexes Bedingungsgefüge äußerer Willkür, innerer Selbstverhältnisse und genuin sozialer Weltbeziehungen (die selbst wiederum eine bestimmte Qualität aufweisen müssen, damit sie die äußere und innere Freiheit tragen können).

Warum ist dieses Freiheitsverständnis in ethischer Hinsicht attraktiv? Der entscheidende Vorteil der hegelschen Konzeption ist ihr Facettenreichtum. Sie erlaubt es nämlich, genau die normativen Maßstäbe, die in der Regel gegeneinander gestellt oder willkürlich addiert werden, als interne Differenzierungen eines einzigen Maßstabs zu verstehen.

1. Während Freiheit und Gleichheit häufig als Kontradiktionen behandelt werden mehr Freiheit führe zu weniger Gleichheit und vice versa -, verdeutlicht Hegel mit seinen Ausführungen zum abstrakten Recht, dass recht verstandene Freiheit nicht nur formale Gleichheit (vor dem Gesetz) impliziere, sondern auch der sozialen Ungleichheit enge Grenzen zu setzen habe. Zwar lasse sich für das Eigentum, welches eine Willensbeziehung zwischen einer Person und einer Sache sei, keine absolute Obergrenze angeben - ,Was und wieviel Ich besitze, ist [...] eine rechtliche Zufälligkeit“ (Hegel 2013, 66) -, jedoch sei der mögliche Eigentumsumfang 
faktisch schon dadurch limitiert, dass eine Willensbeziehung durch Gebrauch regelmäßig zu aktualisieren sei, weshalb es für Hegel auch ein Unrecht darstellt, wenn eine soziale Gruppe ein Eigentumsrecht an Gegenständen geltend macht, die sie selbst gar nicht benutzt, sondern dauerhaft durch andere Personen bearbeiten lässt. ,[W]enn aber nur jener konkrete Besitz mein sein und einem anderen das Eigentum als das Ideelle, Wesentliche zukommen soll, so ist dies eine leere Unterscheidung und eine bloß abstrakte Herrschaft desselben nicht über Sachen, sondern gegen mich“ (Hegel 1983b, 27). Zwar sind diese und ähnliche Passagen im abstrakten Recht noch primär gegen das feudale Eigentumsrecht gerichtet, jedoch lassen sie sich auf die moderne Trennung in Lohnarbeit und Kapital übertragen: Denn obgleich die Mitglieder der bürgerlichen Gesellschaft rechtlich frei und gleich sind, so arbeiten hier doch Personen (Lohnarbeiter) an und mit Gegenständen, ohne in der Regel ein Eigentum an ihnen zu erwerben, während andere Personen (Kapitalisten) Eigentümer an Gegenständen sind und bleiben, ohne diese zu gebrauchen. ,[E]s soll jeder so viel er konsumirt auch produziren. [...] In der Staatswirtschaft sind daher die blossen Konsumenten sehr schlecht angeschrieben, die Kapitalisten, die Hummeln der Gesellschaft, sie sind nicht produktiv, bringen nicht Mittel heran für die Anderen, sie haben diese Mittel, bringen aber keine hervor. Die Arbeit soll daher in dieser Gegenseitigkeit bestehen, die Mittel zur Befriedigung von Bedürfnissen hervorzubringen und diese sollen wieder eine Quelle von Arbeit sein.“ (Hegel 1974, 499). Lohnarbeit war für Hegel weder eine Selbstverständlichkeit noch etwas Erstrebenswertes. In seiner Theorie der bürgerlichen Gesellschaft thematisiert er sie als eine vom Kapitalisten ausnutzbare Notlage, als eine Situation, in der man unselbständig und verwundbar ist. ${ }^{9}$ Positiv orientiert er sich stattdessen am Modell des genossenschaftlich organisierten Handwerksmeisters, dessen Standesehre ein rücksichtsloses Gewinnstreben nicht zulässt. Der Meister beschäftigt deshalb auch nicht etwa Lohnarbeiter (um Kapital zu akkumulieren), sondern er arbeitet zusammen mit seinen Gesellen, die durch ihre Lehre befähigt werden sollen, später einen eigenen Meisterbetrieb zu führen; die rein sachlich begründete Asymmetrie von Meister und Geselle soll gerade nicht auf Dauer gestellt, sondern mittelfristig aufgehoben werden. Anders als der distributive Egalitarismus fordert Hegel nicht die gleiche Verteilung von Gütern, sondern er macht sich im Sinne des deutlich anspruchsvolleren relationalen Egalitarismus für soziale Institutionen stark, durch die nicht zu rechtfertigende Abhängigkeitsund Dominanzverhältnisse in Beziehungen überführt werden, in denen sich die Akteure auf Augenhöhe begegnen können (zur Unterscheidung von distributiver und relationaler Gleichheit siehe Anderson 2012).

2. Damit der Geselle die Meisterprüfung ablegen kann, muss er sich theoretisch wie praktisch bilden. Freiheit hat perfektionistische Implikationen. Sie schließt ein, gattungsspezifische wie individuelle Anlagen entfalten zu können. Dafür bedarf es institutioneller Rahmenbedingungen - und es kann kein Zweifel daran bestehen, dass Klassiker der kritischen Gesellschaftstheorie wie Marx, Fromm, Horkheimer, Adorno und Marcuse den Kapitalismus nicht zuletzt deshalb ablehnten, weil er die freie Entwicklung eines jeden entweder hemme oder Selbstentfaltung

\footnotetext{
${ }^{9}$ Siehe hierzu Ellmers (2015, 67-71).
} 
als Mittel zum Zweck des ökonomischen Wettbewerbs degradiere. In diesem Sinne argumentiert aktuell auch Christoph Henning (2017) für das flourishing als den ethischen Leitwert kritischer Theorie. Gegen konkurrierende Werte wie Freiheit und Gleichheit macht er geltend, dass sie nur bedingt gut seien. Dies zeige sich schon daran, dass es nicht nur zu wenig, sondern auch zu viel Freiheit und Gleichheit geben könne - der Wert beider Normen sei daher abhängig von der Zuträglichkeit zur individuellen Entfaltung. Diese Argumentation kann jedoch nicht überzeugen. Erstens reduziert sie Freiheit auf negative Freiheit. Negative Freiheit, so lässt sich (nicht nur) mit Hegel zeigen, kann jedoch nur ein Aspekt von Freiheit und niemals das Ganze der Freiheit sein. Zweitens lässt sich das quantitative Argument umstandslos auf die Perfektion selbst übertragen: Wie Phänomene der Selbstüberforderung und Rastlosigkeit belegen, kann die Arbeit an den eigenen Talenten ebenfalls Überhand nehmen. Drittens stellt sich die Frage nach der ethischen Qualität dessen, was beim Individuum überhaupt zur Entfaltung gebracht wird. Soll das Ausbilden der Fähigkeiten etwa selbst schon das Gute sein? Dass das Gute im Blühen aufgeht, ist in hohem Maße kontraintuitiv. Nicht die Freiheit hat sich an der Selbstverwirklichung zu relativieren, sondern die Entfaltung der Anlagen als ein freiheitlicher Teilaspekt relativiert sich an den weiteren normativen Implikationen der Freiheit. ${ }^{10}$

3. Am Paradigma des Meisters lässt sich schließlich verdeutlichen, dass recht verstandene Freiheit abgesehen von ihren egalitären und perfektionistischen Aspekten auch eine gemeinschaftliche Dimension aufweist, die von kapitalistischen Gesellschaften systematisch untergraben wird. Die Leitung eines kapitalistischen Konzerns ist zunächst einmal nur dem Konzern selbst gegenüber verpflichtet. Zwar können kapitalistische Betriebe privatrechtliche Assoziationen gründen, um so ihre komplementären Einzelinteressen effektiver zu verfolgen, jedoch unterliegen diese Vereine dem Kartellrecht. Es ist ihnen strikt untersagt, den Wettbewerbsmechanismus substantiell einzuschränken (z. B. durch Preisabsprachen). Anders verhält es sich mit dem von Hegel beschriebenen Meister. Dieser ist Mitglied einer Berufsgenossenschaft, der sogenannten Korporation. Ihre Aufgabe ist es, das ökonomische Kalkül, dem die bürgerliche Gesellschaft zunächst allein verpflichtet zu sein scheint, eine gemeinwohlorientierte Richtung zu geben. Die Berufsgenossenschaft ist nicht nur organisationsrechtlich anders verfasst als der private Verein (der Eintritt beispielsweise ist nicht freiwillig), sie leistet auch aus Sicht ihrer Mitglieder mehr als nur die branchenspezifische Bündelung von Profitinteressen. Erstens sichert sie die Subsistenz ihrer Mitglieder ex ante, indem sie das eigene Gewerbe quantitativ wie qualitativ steuert (durch Zulassungsbeschränkungen und die Standards guter Arbeit). Zweitens sichert sie die Subsistenz ihrer Mitglieder ex post, indem sie im Bedarfsfall Hilfsleistungen organisiert. Diese wechselseitige Unterstützung beruht drittens auf der wechselseitigen Anerkennung und emotio-

\footnotetext{
10 Eine solch interne Selbstrelativierung kennt der Perfektionismus als solcher hingegen nicht. Man kann dies schon daran erkennen, dass er philosophiegeschichtlich sowohl in einer egalitär-liberalen als auch in einer elitär-konservativen Variante auftritt. Die Gründe, die Christoph Henning zufolge für einen egalitärliberalen Perfektionismus sprechen (vgl. Henning 2009, 848-850), sind denn auch keine Implikation des Perfektionismus selbst, sondern externe sozialtheoretische Prämissen oder externe ethische Prinzipien.
} 
nalen Verbundenheit der Genossen, die nicht zuletzt daraus resultieren, dass man dieselbe Berufung teilt, in der korporativen Selbstverwaltung zusammenarbeitet und gemeinsame Aktivitäten unternimmt (wie Feierlichkeiten), die keinen Zweck jenseits der Gemeinschaftlichkeit selbst haben (soziale Praxis im Unterschied zu poietischen Handlungen). Wie in jeder sittlichen Institution führt man auch in der Korporation ein „Leben im Allgemeinen für das Allgemeine“ (Hegel 1983b, 113).

Hegel formuliert seine Theorie der bürgerlichen Gesellschaft an der Schwelle zweier Epochen. Es ist dieser sozial-, wirtschafts- und ideengeschichtlich einzigartige Entstehungskontext, der in Hegels Wirtschaftsphilosophie einen Ausdruck findet, durch den die erst im Entstehen begriffene Moderne zum ersten Mal reflexiv wird: So liegt der korporativen Innensteuerung des Gewerbes die Idee zugrunde, Elemente der alten Wirtschaftsordnung zu reformieren, damit die neuen ökonomischen Verhältnisse, die sich damals erst in wenigen Sektoren abzeichneten, nicht ihr selbstdestruktives, sondern ihr freiheitserweiterndes Potential entfalten. Obgleich es seine Zeit ist, die Hegel in Gedanken fasst - weshalb es verwunderlich wäre, wenn wir 200 Jahre später zu denselben institutionellen Antworten gelangten -, so besteht der bleibende Wert der hegelschen Freiheitslehre doch darin, dass sie mit ihren relational-egalitären, perfektionistischen und kommunalen Implikationen genau die normativen Maßstäbe unter einem Dach zusammenbringt, die auch heute Gesellschaftskritik evozieren.

Funding Open Access funding provided by Projekt DEAL.

Open Access Dieser Artikel wird unter der Creative Commons Namensnennung 4.0 International Lizenz veröffentlicht, welche die Nutzung, Vervielfältigung, Bearbeitung, Verbreitung und Wiedergabe in jeglichem Medium und Format erlaubt, sofern Sie den/die ursprünglichen Autor(en) und die Quelle ordnungsgemäß nennen, einen Link zur Creative Commons Lizenz beifügen und angeben, ob Änderungen vorgenommen wurden.

Die in diesem Artikel enthaltenen Bilder und sonstiges Drittmaterial unterliegen ebenfalls der genannten Creative Commons Lizenz, sofern sich aus der Abbildungslegende nichts anderes ergibt. Sofern das betreffende Material nicht unter der genannten Creative Commons Lizenz steht und die betreffende Handlung nicht nach gesetzlichen Vorschriften erlaubt ist, ist für die oben aufgeführten Weiterverwendungen des Materials die Einwilligung des jeweiligen Rechteinhabers einzuholen.

Weitere Details zur Lizenz entnehmen Sie bitte der Lizenzinformation auf http://creativecommons.org/ licenses/by/4.0/deed.de.

\section{Literatur}

Abicht, Johann Heinrich. 1789/1975. Über die Freiheit des Willens. In Materialien zu Kants ,Kritik der praktischen Vernunft', Hrsg. Rüdiger Bittner und Konrad Kramer, 229-240. Frankfurt am Main: Suhrkamp.

Adorno, Theodor W. 1997a. Freizeit. In Gesammelte Schriften, Band 10.2, Frankfurt am Main: Suhrkamp. Adorno, Theodor W. 1997b. Negative Dialektik. In Gesammelte Schriften, Band 6, Frankfurt am Main: Suhrkamp.

Anderson, Elizabeth S. 2012. Equality. In Oxford Handbook in Political Philosophy, Hrsg. David Estlund, 40-57, Oxford: Oxford University Press. 
Beckermann, Ansgar. 2015. Willensfreiheit. In Handbuch Philosophie und Ethik, Band 2: Disziplinen und Themen, Hrsg. Julian Nida-Rümelin, Irina Spiegel, Markus Tiedemann, 131-140. Paderborn: Ferdinand Schöningh.

Creuzer, Leonhard. 1793/1975. Skeptische Betrachtungen über die Freiheit des Willens. In Materialien zu Kants ,Kritik der praktischen Vernunft', Hrsg. Rüdiger Bittner und Konrad Kramer, 275-294. Frankfurt am Main: Suhrkamp.

Ellmers, Sven. 2015. Freiheit und Wirtschaft. Theorie der bürgerlichen Gesellschaft nach Hegel. Bielefeld: transcript.

Frankfurt, Harry G. 1971. Freedom of the Will and the Concept of a Person. In The Journal of Philosophy 68: 5-20.

Freyenhagen, Fabian. 2013. Adorno's Practical Philosophy. Living Less Wrongly, Cambridge 2013: Cambridge University Press.

Freyenhagen, Fabian. 2017. ,Aber was das Unmenschliche ist, das wissen wir sehr genau'. Zur Normativitätsproblematik bei Adorno. In Warum Kritik? Begründungsformen kritischer Theorien, Hrsg. Sven Ellmers und Philip Hogh, 229-257. Weilerswist: Velbrück.

Guckes, Barbara. 2001. Willensfreiheit trotz Ermangelung einer Alternative? Harry G. Frankfurts hierarchisches Modell des Wünschens. In Freiheit und Selbstbestimmung. Ausgewählte Texte, Hrsg. Monika Betzler und Barbara Guckes, 1-17. Berlin: Akademie Verlag.

Habermas, Jürgen. 1985. Die Verschlingung von Mythos und Aufklärung: Horkheimer und Adorno. In Der philosophische Diskurs der Moderne. Zwölf Vorlesungen, 130-156. Frankfurt am Main: Suhrkamp.

Habermas, Jürgen. 1990. Strukturwandel der Öffentlichkeit. Frankfurt am Main: Suhrkamp.

Hegel, Georg Wilhelm Friedrich. 1970. Grundlinien der Philosophie des Rechts oder Naturrecht und Staatswissenschaft im Grundrisse. Mit Hegels eigenhändigen Notizen und den mündlichen Zusätzen. In: Werke, Band 7. Frankfurt am Main: Suhrkamp.

Hegel, Georg Wilhelm Friedrich. 1974. Philosophie des Rechts. Nach der Vorlesungsnachschrift von K.G.v. Griesheim 1824/25. In: Vorlesungen über Rechtsphilosophie. 1818-1831, Bd. 4, StuttgartBad Cannstatt 1974.

Hegel, Georg Wilhelm Friedrich. 1983a. Philosophie des Rechts. Die Vorlesung von 1819/20 in einer Nachschrift. Frankfurt am Main: Suhrkamp.

Hegel, Georg Wilhelm Friedrich. 1983b. Vorlesungen über Naturrecht und Staatswissenschaft. Heidelberg 1817/18 mit Nachträgen aus der Vorlesung 1818/19. Nachgeschrieben von P. Wannenmann. In: Vorlesungen. Ausgewählte Nachschriften und Manuskripte, Band 1. Hamburg: Meiner.

Hegel, Georg Wilhelm Friedrich. 2005. Die Philosophie des Rechts. Vorlesung von 1821/22. Frankfurt am Main: Suhrkamp.

Hegel, Georg Wilhelm Friedrich. 2013. Grundlinien der Philosophie des Rechts. Hamburg: Meiner.

Henning, Christoph. 2009. Perfektionismus und liberaler Egalitarismus. Ein Versuch ihrer Vermittlung. In DZPhil 57: 845-860. https://doi.org/10.1524/dzph.2009.57.6.845

Henning, Christoph. 2017. Perfektionismus als kritische Theorie. In Warum Kritik? Begründungsformen kritischer Theorien, Hrsg. Sven Ellmers und Philip Hogh, 51-72. Weilerswist: Velbrück.

Höffe, Otfried. 2008. Einführung in die utilitaristische Ethik. Tübingen: Narr Francke Attempto.

Honneth, Axel. 2000. Die soziale Dynamik von Mißachtung. Zur Ortsbestimmung einer kritischen Gesellschaftstheorie. In Das Andere der Gerechtigkeit. Aufsätze zur praktischen Philosophie. Frankfurt am Main: Suhrkamp.

Honneth, Axel. 2001. Leiden an Unbestimmtheit. Eine Reaktualisierung der Hegelschen Rechtsphilosophie. Stuttgart: Reclam.

Honneth, Axel. 2011. Das Recht der Freiheit. Grundriß einer demokratischen Sittlichkeit. Berlin: Suhrkamp.

Honneth, Axel. 2015. Die Idee des Sozialismus. Berlin: Suhrkamp.

Jaeggi, Rahel. 2014. Kritik von Lebensformen. Berlin: Suhrkamp.

Kant, Immanuel. 1794/1907. Die Religion innerhalb der Grenzen der bloßen Vernunft. In Akademieausgabe, Hrsg. Königlich Preußische Akademie der Wissenschaften, Band VIII. Berlin: de Gruyter.

Kant, Immanuel. 1797/1907. Metaphysik der Sitten. In Akademieausgabe, Hrsg. Königlich Preußische Akademie der Wissenschaften, Band VI. Berlin: Reimer.

Kant, Immanuel. 1788/1913. Kritik der praktischen Vernunft. In Akademieausgabe, Hrsg. Königlich PreuBische Akademie der Wissenschaften, Band V. Berlin: Reimer.

Kant, Immanuel. 1795/1923. Zum ewigen Frieden. In Akademieausgabe, Hrsg. Königlich Preußische Akademie der Wissenschaften, Band VIII. Berlin: de Gruyter.

Kersting, Wolfgang. 2000. Theorien sozialer Gerechtigkeit. Stuttgart: J.B. Metzler. 
Klar, Samuel. 2007. Moral und Politik bei Kant. Eine Untersuchung zu Kants praktischer und politischer Philosophie im Ausgang der „Religion innerhalb der Grenzen der bloßen Vernunft“. Würzburg: Königshausen \& Neumann.

Knappik, Franz. 2013. Im Reich der Freiheit. Hegels Theorie autonomer Vernunft. Berlin/Boston: De Gruyter.

Kuhne, Frank. 2015. Transformation der praktischen Philosophie in kritische Theorie der Gesellschaft. Zum Problem der normativen Grundlage des Kapitals und der Kritischen Theorie. In Zeitschrift für kritische Sozialtheorie und Philosophie 2 (1): 139-170. https://doi.org/10.1515/zksp-2015-0007

Kuhne, Frank. 2017. Moral im „Kapital“"? Hat Marx’ Kritik der politischen Ökonomie normative Grundlagen? In Warum Kritik? Begründungsformen kritischer Theorien, Hrsg. Sven Ellmers und Philip Hogh, 190-209. Weilerswist: Velbrück.

Lumer, Christop. 1997. Habermas' Diskursethik. In Zeitschrift für philosophische Forschung 51: 42-64.

MacIntyre, Alasdair. 1995. Der Verlust der Tugend. Zur moralischen Krise der Gegenwart. Frankfurt am Main: Suhrkamp.

Marx, Karl. 2001. Das Kapital. Kritik der politischen Ökonomie. In Marx-Engels-Werke, Bd. 23. Berlin: Dietz-Verlag.

Pippin, Robert. 2005. Hegel und das Problem der Freiheit. In Die Verwirklichung der Freiheit. Der Idealismus als Diskurs der Moderne, 59-70. Frankfurt am Main: Campus.

Quante, Michael. 1993. Hegels Begriff der Handlung. Stuttgart-Bad Cannstaat: Frommann-Holzboog.

Quante, Michael. 2011. Die Wirklichkeit des Geistes. Studien zu Hegel. Berlin: Suhrkamp.

Rawls, John. 2001. Justice as Fairness: A Restatement. Cambridge MA: Harvard University Press.

Reinhold, Carl Leonhard. 1792/1975. Erörterung des Begriffs von der Freiheit des Willens. In Materialien zu Kants' ,Kritik der praktischen Vernunft', Hrsg. Rüdiger Bittner und Konrad Kramer, 252-274. Frankfurt am Main: Suhrkamp.

Rosa, Hartmut. 2009. Kapitalismus als Dynamisierungsspirale - Soziologie als Gesellschaftskritik. In Soziologie - Kapitalismus - Kritik. Eine Debatte, Hrsg. Klaus Dörre, Stephan Lessenich, Hartmut Rosa, 87-125. Frankfurt am Main: Suhrkamp.

Rosa, Hartmut. 2011. Terrorists and High-speed Surfers: Towards a Sociological Conception of Performative Identity. In: BIOS - Zeitschrift für Biographieforschung, Oral History und Lebensverlaufsanalysen 24: 204-223.

Rosa, Hartmut. 2012. Weltbeziehungen im Zeitalter der Beschleunigung. Berlin: Suhrkamp.

Rosa, Hartmut. 2017. Eskalation oder Ausweg? Das Ende der dynamischen Stabilisierung und das Konzept der Resonanz. In Warum Kritik? Begründungsformen kritischer Theorien, Hrsg. Sven Ellmers und Philip Hogh, 339-363. Weilerswist: Velbrück.

Ruschig, Ulrich. 2010. Kant und Marx. In Von der Hermeneutik zur interkulturellen Philosophie. Festschrift für Heinz Kimmerle zum 80. Geburtstag, Hrsg. Hamid Reza Yousefi, Hermann-Josef Scheidgen und Henk Oosterling, 211-224. Nordhausen: bautz.

Stahl, Titus. 2013. Immanente Kritik. Elemente einer Theorie sozialer Praktiken. Frankfurt am Main: Campus.

Steinhoff, Uwe. 2006. Kritik der kommunikativen Rationalität: Eine Darstellung und Kritik der kommunikationstheoretischen Philosophie von Jürgen Habermas und Karl-Otto Apel. Paderborn: Mentis.

Stemmer, Peter. 2000. Handeln zugunsten anderer. Eine moralphilosophische Untersuchung. Berlin/New York: Walter de Gruyter.

Zunke, Christine. 2011. Es gibt nur einen vernünftigen Grund, Freiheit gesellschaftlich verwirklichen zu wollen: Moral. In Die Moral in der Kritik. Ethik als Grundlage und Gegenstand kritischer Gesellschaftstheorie, Hrsg. Sven Ellmers und Ingo Elbe, 11-37. Würzburg: Königshausen \& Neumann. 